\title{
Diagnosis of Multiple Scan Chain Timing Faults
}

\author{
Wei-Shun Chuang, Shiu-Ting Lin, Wei-Chih Liu, and James Chien-Mo Li, Member, IEEE
}

\begin{abstract}
A diagnosis technique is presented to locate multiple timing faults in scan chains. Jump Simulation is a novel parallel simulation technique which quickly searches for the upper and the lower bounds of every individual fault. The proposed technique takes into account the interaction of multiple faults so the diagnosis results are deterministic, not probabilistic. This technique is very useful in the production test environment because it requires only regular automated test pattern generator patterns, not specialized diagnosis patterns. Experiments on ISCAS'89 benchmark circuits show that this technique can successfully pinpoint almost every single one of $\mathbf{1 6}$ hold-time faults in a scan chain of more than 800 scan cells. The proposed technique is still effective when failure data are limited or faults are clustered.
\end{abstract}

Index Terms-Automated test pattern generator (ATPG), fault diagnosis, scan-based test, silicon debug.

\section{INTRODUCTION}

$\mathbf{S}$ CAN CHAINS can occupy up to $30 \%$ of the silicon area [13], so the yield loss caused by defective scan chains cannot be underestimated. Many real chips with defective scan chains have recently been reported by the industry [3], [5], [7], [22], [23]. In nanometer technologies, systematic defects and process variation have become a more challenging issue than random defects [9], [10]. Systematic defects and process variation in scan cells can manifest themselves as multiple scan chain timing faults. Therefore, a software tool that diagnoses multiple scan chain faults is very much needed to identify the root causes and shorten the time to market.

Fig. 1 shows a faulty scan chain with a hold-time (HT) fault in scan cell 2. The scan cells are indexed in descending order, from the scan input (SI) to the scan output (SO). The length of the scan chain $(L)$ is the total number of scan cells in the chain. A scan cell can be either a scanable flip-flop or latch. In this figure, the length of scan chain is five $(L=5)$, and the scan cells are indexed from four to zero. Those scan cells whose indexes are larger than $i$ are upstream to scan cell $i$. Those scan cells whose indexes are smaller than $i$ are downstream to scan cell $i$. For example, scan cells 1 and 0 are downstream to scan cell 2; scan cells 3 and 4 are upstream to scan cell 2. When the SI pattern (good SI) is scanned into the faulty chain, the transition downstream to scan cell 2 is flipped (underlined). When the SO pattern (expected SO) is scanned out of the faulty chain, the transition upstream to scan cell 2 is flipped.

Manuscript received August 20, 2007; revised December 4, 2007. This paper was recommended by Associate Editor K. Chakrabarty.

W.-S. Chuang is with Springsoft, Inc., Hsinchu 30075, Taiwan, R.O.C.

S.-T. Lin, W.-C. Liu, and J. C.-M. Li are with the Graduate Institute of Electronics Engineering, National Taiwan University, Taipei 10617, Taiwan, R.O.C. (e-mail: cmli@cc.ee.ntu.edu.tw).

Digital Object Identifier 10.1109/TCAD.2008.923258

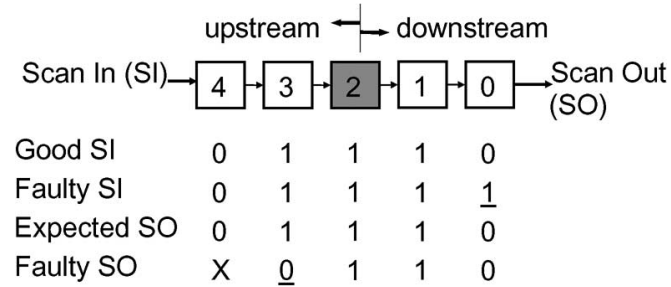

Fig. 1. HT fault in scan cell 2.

Diagnosis of single scan chain fault has been under research for more than ten years and is becoming available in commercial tools and patents, such as [2], [5], [7], and [27]. However, multiple timing fault diagnosis is still a challenging issue. Assuming a faulty scan chain with ten faults in 1000 scan cells, there are, approximately, $10^{30}$ combinations of fault locations. It is infeasible to exhaustively enumerate all combinations, so a smart diagnosis algorithm has to be developed to diagnose multiple faults.

Multiple scan chain timing faults can be attributed to three categories of problems: defective scan cells, incorrect timing, and clock skew. For the first category, defective transistors (such as leakage and incorrect implants) and highly resistive bridging defects are shown to be culprits for setup-time (ST) faults [19] and HT faults [12], respectively. These defects, when located on the scan path, affect only the scan operation but not the normal operation. For the second category, signal integrity problems (such as crosstalk) can cause timing faults [5]. Multiple ST faults are likely to occur when the automatic router gives priority to functional signals over the scan signals. Also, HT faults can be caused by HT violations due to insufficient buffers or timing variation. For the third category, clock skew can result from design error, process variation, or systematic defects. HT fault occurs in cell $i$ if the active clock edge arrives scan cell $i+1$ much earlier than it arrives scan cell $i$. Based on the above description, it can be concluded that some ST faults (such as routing congestion) can be eliminated by slow speed shifting but the other ST faults cannot. Although slow shifting is effective, fault diagnosis is still important for failure analysis to identify the root causes. Similarly, some HT faults (such as clock skew) may be fixed by adopting the LSSD design, but the other HT faults may not. Even if some HT faults can be eliminated by changing the scan design, it is still critical to diagnose those chips that are already manufactured for yield improvement.

Jump Simulation is a fast diagnosis technique that searches for upper and lower bounds of scan chain faults by parallel simulation [8]. The Jump Simulation diagnosis is based on the "proof by contradiction" principle. The technique first assumes multiple hypothetical bounds and then performs parallel simulation to proof incorrect bounds. Although it is very effective 
TABLE I

Excitation Condition of Six Fault Models

\begin{tabular}{|l|c|c|c|}
\hline $\begin{array}{c}\text { Fault } \\
\text { Model }\end{array}$ & $\begin{array}{c}\text { previous } \\
\text { cycle }\end{array}$ & $\begin{array}{c}\text { current } \\
\text { cycle }\end{array}$ & $\begin{array}{c}\text { next } \\
\text { cycle }\end{array}$ \\
\hline STR & 0 & 1 & - \\
\hline STF & 1 & 0 & - \\
\hline \multirow{2}{*}{ ST } & 1 & 0 & - \\
\cline { 2 - 4 } & 0 & 1 & - \\
\hline FTR & - & 0 & 1 \\
\hline FTF & - & 1 & 0 \\
\hline \multirow{2}{*}{ HT } & - & 1 & 0 \\
\cline { 2 - 4 } & - & 0 & 1 \\
\hline
\end{tabular}

for single faults, the previous version of Jump Simulation cannot deal with multiple faults. This paper further develops the concept of Jump Simulation so that it can now be used to diagnose multiple timing faults. This technique supports six timing fault models that were used in previous research: slow-to-rise (STR), slow-to-fall (STF), ST, fast-to-rise (FTR), fast-to-fall (FTF), and holt-time faults. This diagnosis produces deterministic results which are valid although multiple faults interact with each other. Experimental results on ISCAS'89 benchmark circuits show that the proposed technique is able to pinpoint almost every one of $16 \mathrm{HT}$ faults in a faulty scan chain. This diagnosis technique does not need specialized diagnosis patterns - just regular automated test pattern generator (ATPG) patterns are enough. The proposed technique is still effective even when the failure data are truncated due to limited automatic test equipment (ATE) memory. These features altogether make the proposed technique very useful in the production test environment.

The organization of this paper is as follows. Section II introduces fault models, terminology, assumption, and past research in this area. Section III presents the Jump Simulation diagnosis technique. Section IV shows experimental results, and Section $\mathrm{V}$ discusses issues related to the proposed technique. Finally, Section VI summarizes this paper.

\section{BACKGROUND}

\section{A. Scan Chain Timing Fault Model}

When the faulty scan chain is operated in shift mode, the actual value of a faulty cell is flipped when certain excitation conditions are met. The excitation conditions of five scan chain timing fault models are listed in Table I. An STR fault is excited when the faulty scan cell is expected to have a rising transition, i.e., previous cycle is zero, and the current cycle is one. The fault effect is that the faulty scan cell remains zero for the current cycle. An STF fault is excited by a falling transition, and the faulty scan cell remains one for the current cycle. The ST fault is a combination of STF and STR faults. Both the rising and the falling transition are delayed by a clock cycle. An FTR fault is excited when the faulty scan cell is expected to rise in the next cycle, i.e., current cycle is zero, and the next cycle is one. The fault effect is that the faulty scan cell rises one cycle earlier than expected. An FTF fault is excited when the faulty scan cell is expected to fall in the next cycle. The HT fault is excited by either a falling or a rising transition. The HT fault is a combination of FTR and FTF faults. The HT faulty scan cell can be regarded as a buffer which scan data pass through.

Fig. 1 shows an example where scan cell 2 has an HT fault. After the scan-in operation, the rising transition downstream to scan cell 2 excites the fault so the value of scan cell 0 is flipped. The good SI is "01110," but the faulty SI is "01111." The underline represents the affected bit which is flipped in the presence of a fault. Similarly, if the expected SO is "01110," the faulty SO turns out to be "X01111." The falling transition upstream to scan cell 2 excites the fault so the value of scan cell 3 is flipped. The actual value of the most upstream bit is uncertain so it is denoted as X.

\section{B. Past Research}

Previous related work is reviewed in two categories: hardware and software. In the hardware category, Schafer et al. [21] proposed to add extra routings from one scan chain to a partner scan chain. The contents of the scan chain under diagnosis (ChUD) can be observed by the partner scan chain. Edirisooriya and Edirisooriya [1] proposed to insert XOR gates into scan chains so that the contents of scan cells can be flipped before shifting into the next scan cell. Narayanan and Das [18] and $\mathrm{Wu}$ [25] proposed to flip the contents of each scan cell by modifying the scan cell design. The area overhead problem makes the aforementioned hardware solutions impractical. What is worse, the hardware solutions are too late for chips that are already designed and manufactured.

In the software diagnosis category, Kundu [13] proposed a sequential ATPG method to generate diagnosis patterns. A sequence of patterns is generated to control every scan cell to the desired value. Kundu's idea requires sequential ATPG and is limited to single stuck-at faults only. Stanley [23] presented a diagnosis tool that performs fault simulations for all latches in the scan chain. Faulty latches are diagnosed if their scores, which represent the degree of similarity between faulty outputs and good outputs, are higher than a certain threshold. Scorebased diagnosis of multiple faults is not guaranteed to be correct because the interaction among faults is not taken into consideration. Hirase et al. [4] presented an $I_{\mathrm{DDQ}}$ diagnosis technique for faults in scan chains. Guo and Venkataraman [2], [3] proposed a three-step diagnosis procedure. In the first step, the faulty chain and the fault model are determined by applying a three-pattern test. In the second step, the upper and the lower bounds of the faulty cell are determined by logic simulations with unknown values in the whole faulty chain. In the third step, every fault between the bounds is exhaustively simulated, and the results are compared with observed outputs. Their technique is very good for single fault but has limitation for multiple faults because the combination of fault locations is too large for exhaustive simulation. Huang et al. [5], [6] proposed a probabilistic model for intermittent timing faults in scan chains. Their technique handles multiple faults by ranking the probability of a group of candidate faults. Besides regular ATPG patterns, scan chain diagnosis patterns have been proposed in [16], [17], [24], and [26]. Although diagnosis patterns provide good results for multiple faults, they are not popular in the production test environment. 
TABLE II

PRoposed TWO-PATTERN TEST

\begin{tabular}{|l|c|c|}
\hline \multicolumn{1}{|c|}{ Pattern } & Pattern 1 & Pattern 2 \\
\hline SI & 11110000 & 00001111 \\
\hline Expected SO & 11110000 & 00001111 \\
\hline Faulty SO (STR) & $11 \underline{000000}$ & 00001111 \\
\hline Faulty SO (STF) & 11110000 & $00 \underline{111111}$ \\
\hline Faulty SO (ST) & 11000000 & $00 \underline{111111}$ \\
\hline Faulty SO (FTR) & $1111 \underline{1100}$ & 00001111 \\
\hline Faulty SO (FTF) & 11110000 & $0000 \underline{0} 11$ \\
\hline Faulty SO (HT) & $1111 \underline{1100}$ & 0000 0011 \\
\hline
\end{tabular}

\section{Determine Fault Model and Number of Faults}

Guo and Venkataraman [2] proposed a three-pattern test to determine the scan chain fault model. The three-pattern test cannot determine the number of faults and, hence, is not good enough for multiple fault diagnosis. Table II shows a twopattern test proposed to determine the fault model and the number of faults [16], [17]. Each pattern is of equal length $L$. In the first pattern, the downstream $L / 2$ bits are all zeros, and the upstream $L / 2$ bits are all ones. The second pattern is the bitwise complement of the first pattern. On the tester, the scan chain is initialized by shifting the first (the rightmost) bit of the first pattern for $L$ times. The first pattern is then scanned in, followed by an immediate scan out without any system clock. The SOs are recorded by the tester without an immediate pass/fail decision. The same procedure is repeated for the second pattern.

The faulty SOs of six timing fault models are shown in Table II, with affected bits underlined. For illustration, this table assumes two faults in the faulty chain. Generally speaking, if there are $N$ STR faults in the chain, there will be $N$ more zeros than expected in the first SO pattern. If there are $N$ FTR faults, there will be $N$ more ones than expected in the first SO pattern. Similarly, STF faults and FTF faults are detected in the second pattern by $N$ more ones and $N$ more zeros than expected, respectively. The number of faults equals the number of bits flipped in the SOs. The maximum number of countable timing faults is half the length of scan chains $L / 2$. In the following text, it is assumed that the fault model and the number of faults $(N)$ are already known.

The scan chains that fail the two-pattern test are faulty; the scan chains that pass both tests are good. During the diagnosis process, the faulty scan chain is called ChUD, and the other scan chains are called chains under no diagnosis (ChUND). For clear explanation, we demonstrate our technique using one single faulty chain in the following text.

\section{Definition}

Assume that there are $N$ faults in the ChUD, and the faults are indexed in a decreasing order $\left(f_{N-1}\right.$, $f_{N-2}, \ldots, f_{n}, \ldots, f_{0}$ ), from SI to SO. Fault $f_{N-1}$ represents the most upstream fault, and $f_{0}$ represents the most downstream fault. The subscript $n$ is referred to as the fault index. The upper bound of fault $f_{n}\left(\mathrm{UB}_{n}\right)$ is the most upstream scan cell, where $f_{n}$ is suspected to be located, including $\mathrm{UB}_{n}$ itself. The lower bound of fault $f_{n}\left(\mathrm{LB}_{n}\right)$ is the most downstream cell where $f_{n}$ is suspected to be located, including $\mathrm{LB}_{n}$ itself. According to this definition, it can be derived that $\mathrm{UB}_{n}$ and $\mathrm{LB}_{n}$ must strictly increase with respect to their fault index. No equal sign is allowed because multiple faults cannot occur in the same scan cell

$$
\begin{aligned}
& \mathrm{UB}_{N-1}>\mathrm{UB}_{N-2}>\cdots>\mathrm{UB}_{0} \\
& \mathrm{LB}_{N-1}>\mathrm{LB}_{N-2}>\cdots>\mathrm{LB}_{0} .
\end{aligned}
$$

Fig. 2 shows an example faulty scan chain of length eighteen $(L=18)$ and five faults $(N=5)$. The first row shows the initial location of bounds before the diagnosis starts. The upper bounds are located in the five most upstream scan cells, in decreasing order of $n$. The lower bounds are located in the five most downstream scan cells, in increasing order of $n$. These initial bounds simply indicate that the five faults can be anywhere in the scan chain.

As diagnosis proceeds, the upper and the lower bounds are tightened for each fault. The second row shows an example configuration of the bounds during diagnosis. Originally, $\mathrm{LB}_{4}$ is 4 but now $\mathrm{LB}_{4}$ is moved to 15 . This means fault $f_{4}$ can be found within only three scan cells: 17,16 , or 15 . The gray rectangles show the interval where each fault can be located. Please note that the upper bound of a fault is always greater than or equal to its own lower bound. That is

$$
\mathrm{UB}_{n} \leq \mathrm{LB}_{n} .
$$

Fault $f_{n}$ is exactly diagnosed when $\mathrm{UB}_{n}$ is equal to $\mathrm{LB}_{n}$. For instance, fault $f_{0}$ in Fig. 2 is exactly diagnosed because both $\mathrm{UB}_{0}$ and $\mathrm{LB}_{0}$ are equal to scan cell 3 . In the presence of multiple faults, it is not always possible to exactly diagnose every fault. Given the bounds in the second row in Fig. 2, there are totally 192 combinations of fault locations. For example, $f_{4}=16, f_{3}=13, f_{2}=10, f_{1}=7, f_{0}=3$ is one possible combination of fault locations. The tighter the bounds become, the fewer combinations of fault locations remain, and the easier it is to search for defects in physical failure analysis.

Fig. 3 depicts the simulation model for our diagnosis technique. There are three components on the input side of this model: primary input (PI), ChUND-SI, and ChUD-SI. The SIs to ChUND and ChUD are denoted as ChUND-SI and ChUDSI, respectively. The PIs and ChUND-SI are directly applied to the circuit as they are. The ChUD-SI filler modifies the SI patterns according to the location of UB and LB. ChUD - $\mathrm{SI}_{\mathrm{b}}$ and ChUD - $\mathrm{SI}_{\mathrm{a}}$ represents the SI patterns before and after the ChUD-SI filler, respectively. SIs upstream to $\mathrm{UB}_{N-1}$ remains unchanged, but SIs downstream to $\mathrm{LB}_{0}$ are definitely affected. How the question marks between $\mathrm{UB}_{N-1}$ and $\mathrm{LB}_{0}$ are filled will be explained in Sections III-B and C. Please note that, although Fig. 3 shows only two HT faults, the simulation model is applicable to $N$ fault diagnosis.

The output side of the simulation model also has three components: primary output (PO), ChUND-SO, and ChUDSO. The SOs of ChUND and ChUD are denoted as ChUNDSO and ChUD-SO, respectively. The POs and ChUND-SO are observed without any modification. The ChUD-SO filler 


\begin{tabular}{|l|c|c|c|c|c|c|c|c|c|c|c|c|c|c|c|c|c|c|}
\hline & 17 & 16 & 15 & 14 & 13 & 12 & 11 & 10 & 9 & 8 & 7 & 6 & 5 & 4 & 3 & 2 & 1 & 0 \\
\hline $\begin{array}{l}\text { before } \\
\text { diagnosis }\end{array}$ & $\mathrm{UB}_{4}$ & $\mathrm{UB}_{3}$ & $\mathrm{UB}_{2}$ & $\mathrm{UB}_{1}$ & $\mathrm{UB}_{0}$ & & & & & & & & & $\mathrm{LB}_{4}$ & $\mathrm{LB}_{3}$ & $\mathrm{LB}_{2}$ & $\mathrm{LB}_{1}$ & $\mathrm{LB}_{0}$ \\
\hline $\begin{array}{l}\text { during } \\
\text { diagnosis }\end{array}$ & $\mathrm{UB}_{4}$ & & $\mathrm{LB}_{4}$ & & $\mathrm{UB}_{3}$ & $\mathrm{UB}_{2}$ & $\mathrm{UB}_{1}$ & & $\mathrm{LB}_{3}$ & & & $\mathrm{LB}_{2}$ & $\mathrm{LB}_{1}$ & & $\begin{array}{c}\mathrm{UB}_{0} \\
\mathrm{LB}_{0}\end{array}$ & & \\
\hline
\end{tabular}

Fig. 2. Example ChUD $(L=18, N=5)$.

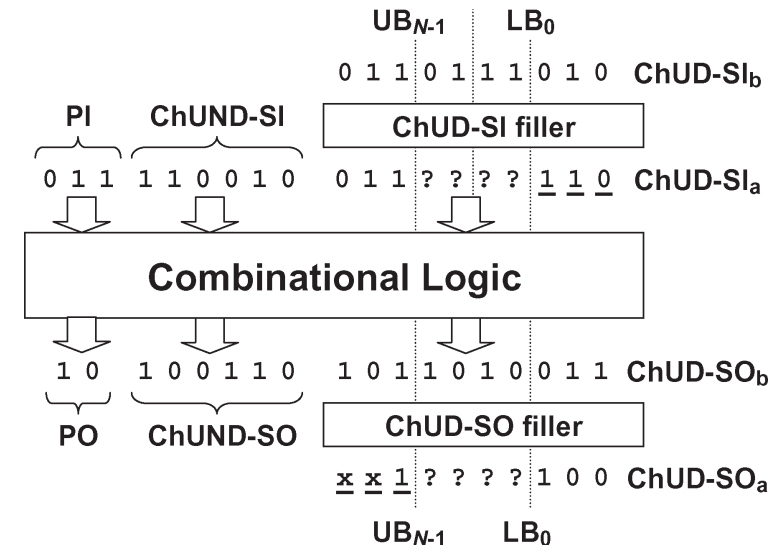

Fig. 3. Simulation model.

modifies the SO patterns according to the location of UB and LB. SOs downstream to $\mathrm{LB}_{0}$ remains unaffected but SOs downstream to $\mathrm{UB}_{N-1}$ are definitely affected. How the question marks between $\mathrm{UB}_{N-1}$ and $\mathrm{LB}_{0}$ are filled will be explained in Section III-D.

\section{E. Assumptions}

This paper is based on the following three assumptions. First, for clear explanation, the following text assumes only one single faulty chain, and there is no inversion in the scan chains. Please see the discussion in Sections V-A and B for the potential extension to handle multiple faulty chains and inversion in the chain. Second, all timing faults in a faulty scan chain belong to the same fault model. This assumption is valid when the faults are caused by the same systematic defect or the same design mistake. Please see Section V-C for detailed discussion. Finally, this technique diagnoses the faults which are consistent and permanent. Although intermittent fault can be detected by our sanity check, accurate diagnosis of intermittent multiple faults is still under research.

\section{Proposed Diagnosis TeChnique}

\section{A. Diagnosis Flow}

Fig. 4 shows the proposed diagnosis flow. The diagnosis is composed of two key components: Bounds Finder and Exhaustive Simulator. The former is a coarse resolution simulator which quickly searches for a pair of upper and lower bounds of each fault. The latter is a fine resolution simulator which exhaustively tries every scan cells between the upper and the lower bounds for each fault.

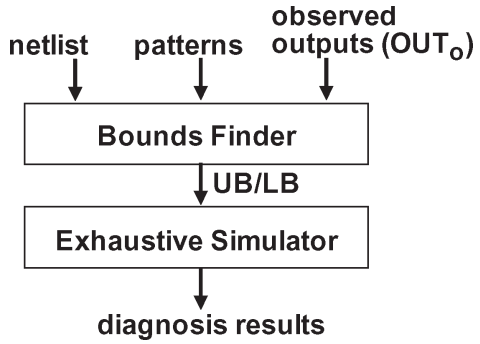

Fig. 4. Overall diagnosis flow.

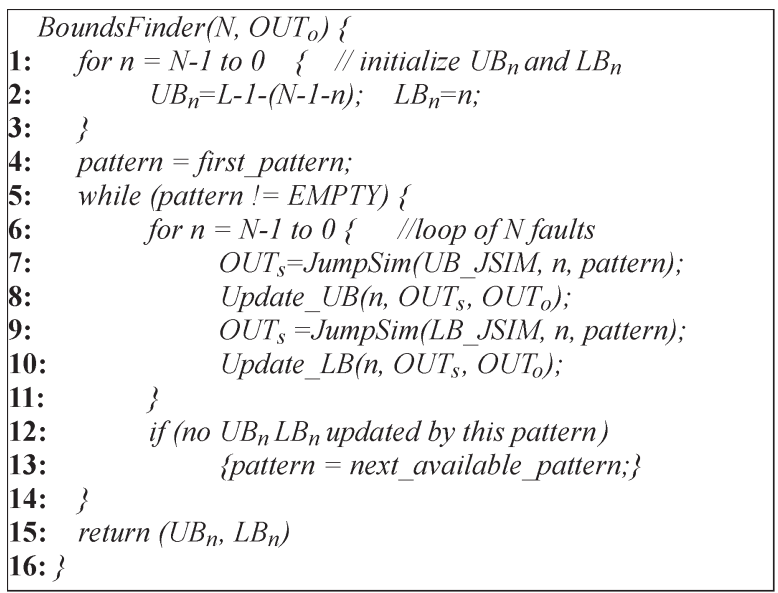

Fig. 5. Bounds Finder algorithm.

Fig. 5 shows the algorithm of Bounds Finder. Every line in the algorithm is explained as follows.

Lines 1-3. Initialize upper and lower bounds. $\mathrm{UB}_{n}$ is placed at the entrance of scan chain in decreasing order of $n . \mathrm{LB}_{n}$ is placed at the exit of scan chain in increasing order of $n$ (see the first row in Fig. 2).

Lines 4-7. For every fault $f_{n}$, one UB Jump Simulation is performed on the first pattern.

Line 8. Simulation outputs $\left(\mathrm{OUT}_{\mathrm{s}}\right)$ are compared with the observed outputs from ATE $\left(\mathrm{OUT}_{\mathrm{O}}\right)$. The upper bounds are updated according to the comparison result.

Lines 9-11. One LB Jump Simulation is performed on the same pattern. $\mathrm{OUT}_{\mathrm{s}}$ are compared with $\mathrm{OUT}_{\mathrm{o}}$, and the $\mathrm{LB}_{n}$ is updated accordingly.

Lines 12-14. If there is any UB or LB tightened by this pattern, repeat the Jump Simulation with the same pattern applied; otherwise, simulate the next pattern. The process repeats until there is no more pattern to simulate. 


\begin{tabular}{|l|c|c|c|c|c|c|c|c|c|c|c|c|c|c|c|c|c|c|}
\hline$i$ & 17 & 16 & 15 & 14 & 13 & 12 & 11 & 10 & 9 & 8 & 7 & 6 & 5 & 4 & 3 & 2 & 1 & 0 \\
\hline$S I[5, \mathrm{i}]$ & 0 & 1 & 1 & 0 & 0 & 0 & 1 & 0 & 0 & 1 & 1 & 0 & 0 & 0 & 1 & 1 & 0 & 0 \\
\hline$S I[4, \mathrm{i}]$ & $\mathrm{X}$ & 0 & 1 & 1 & 0 & 0 & 0 & 1 & 0 & 0 & 1 & 1 & 0 & 0 & - & - & - & - \\
\hline$S I[3, i]$ & - & $\mathrm{X}$ & 0 & 1 & 1 & 0 & 0 & 0 & 1 & 0 & 0 & 1 & 1 & 0 & 0 & - & - & - \\
\hline$S I[2, i]$ & - & - & $\mathrm{X}$ & 0 & 1 & 1 & 0 & 0 & 0 & 1 & 0 & 0 & 1 & 1 & 0 & 0 & - & - \\
\hline$S I[1, i]$ & - & - & - & $\mathrm{X}$ & 0 & 1 & 1 & 0 & 0 & 0 & 1 & 0 & 0 & 1 & 1 & 0 & 0 & - \\
\hline$S I[0, i]$ & - & - & - & - & $\mathrm{X}$ & 0 & 1 & 1 & 0 & 0 & 0 & 1 & 0 & 0 & 1 & 1 & 0 & 0 \\
\hline $\mathrm{UB}$ & $\mathrm{UB}_{4}$ & & & & $\mathrm{UB}_{3}$ & $\mathrm{UB}_{2}$ & $\mathrm{UB}_{1}$ & & & & & & & & $\mathrm{UB}_{0}$ & & & \\
\hline $\mathrm{LB}$ & & & $\mathrm{LB}_{4}$ & & & & & & $\mathrm{LB}_{3}$ & & & $\mathrm{LB}_{2}$ & $\mathrm{LB}_{1}$ & & $\mathrm{LB}_{0}$ & & & \\
\hline $\mathrm{NULB}$ & - & - & 4 & 4 & 4 & 4 & 4 & 4 & 3 & 3 & 3 & 2 & 1 & 1 & 0 & 0 & 0 & 0 \\
\hline NDUB & 3 & 3 & 3 & 3 & 2 & 1 & 0 & 0 & 0 & 0 & 0 & 0 & 0 & 0 & - & - & - & - \\
\hline$j$ & 5 & 5 & 4 & 4 & 4 & 4 & 4 & 4 & 3 & 3 & 3 & 2 & 1 & 1 & 0 & 0 & 0 & 0 \\
\hline$k$ & 4 & 4 & 4 & 4 & 3 & 2 & 1 & 1 & 1 & 1 & 1 & 1 & 1 & 1 & 0 & 0 & 0 & 0 \\
\hline $\begin{array}{l}\text { UB_JSIM } \\
\text { ChUD-SI } \\
n=2 ; j b=10\end{array}$ & $\mathrm{X}$ & $\mathrm{X}$ & 1 & 1 & $\mathrm{X}$ & $\mathrm{X}$ & $\mathrm{X}$ & 0 & 0 & $\mathrm{X}$ & $\mathrm{X}$ & 0 & 0 & 1 & 1 & 1 & 0 & 0 \\
\hline $\begin{array}{l}\mathrm{LB} \_ \text {JSIM } \\
\begin{array}{l}\text { ChUD-SI } \\
n=2 ; j b=10\end{array}\end{array}$ & $\mathrm{X}$ & $\mathrm{X}$ & 1 & 1 & $\mathrm{X}$ & 0 & 0 & $\mathrm{X}$ & $\mathrm{X}$ & $\mathrm{X}$ & $\mathrm{X}$ & 0 & 0 & 1 & 1 & 1 & 0 & 0 \\
\hline
\end{tabular}

Fig. 6. Example to illustrate ChUD-SI filling.

\section{B. SI Matrix}

Before explaining the Bounds Finder algorithm, it is essential to understand what values are loaded into the faulty chain in the presence of multiple faults. After the scan-in operation, the faulty values of ChUD-SI can be represented by an SI matrix. The SI matrix has $N+1$ rows and $L$ columns. Every row represents a fault index $(n)$, and every column represents a scan cell $(i)$. The value of $\operatorname{SI}[n, i]$ is the value of scan cell $i$ when cell $i$ is downstream to $f_{n}$ (inclusive) but upstream to $f_{n-1}$ (exclusive). Fig. 6 shows an example of the SI matrix of HT fault $(N=5)$. The first row SI $[5, i]$ shows the good SI values without being affected by any fault (i.e., $\mathrm{ChUD}-\mathrm{SI}_{\mathrm{b}}$ in Fig. 3). The second row $\mathrm{SI}[4, i]$ shows the values when cell $i$ is downstream to $f_{4}$ but upstream to $f_{3}$. Similarly, the remaining four rows $\operatorname{SI}[3, i]$ to $\operatorname{SI}[0, i]$ can be viewed in the same way. Every row in the SI matrix is the right shifted version of its preceding row. The symbol " $\mathrm{X}$ " in the SI matrix indicates that the value is dependent on the next SI. The symbol "-" in the SI matrix represents a don't care which can never occur. For example, it is impossible to have two faults upstream to scan cell 17 so $\mathrm{SI}[3,17]$ is marked as "-." The SI matrix for the other fault models can be derived in the similar way.

Given the upper and the lower bounds, the potential values of every scan cell are highlighted in gray in the SI matrix in Fig. 6 . The potential value of a scan cell $i$ form a continuous segment in the SI matrix, from $\operatorname{SI}[j, i]$ to $\operatorname{SI}[k, i]$. Let $j$ and $k$ denote the maximum fault index and the minimum fault index, respectively. Because scan cell 16 is between $\mathrm{UB}_{4}$ and $\mathrm{LB}_{4}$, it has two potential values: $\operatorname{SI}[5,16]$ and $\operatorname{SI}[4,16]$. If $f_{4}$ is located in cell 16 or 17, then the value of scan cell 16 would be zero. Otherwise, if $f_{4}$ is located in scan cell 15 , then the value of scan cell 16 would be one. Hence, for scan cell $16, j$ is equal to five and $k$ is equal to four. For another example, scan cell 10 has four potential values, from $\operatorname{SI}[4,10]$ to $\operatorname{SI}[1,10]$, so $j=4$, $k=1$.

The values of $j$ and $k$ can be determined by the nearest upstream LB (NULB) and the nearest downstream UB (NDUB) of a scan cell. The former is the fault index of the closest LB upstream to cell $i$, including cell $i$ itself. The latter is the fault index of the closest UB downstream to cell $i$, excluding cell $i$ itself. For scan cell 10, its NULB is four $\left(\mathrm{LB}_{4}=\right.$ cell 15$)$, and NDUB is zero $\left(\mathrm{UB}_{0}=\right.$ cell 3$)$. For scan cell 16 , its NDUB is three, but its NULB is not available, which is denoted by a dash sign. Equation (4) states that, if NULB of scan cell $i$ is available, then $j$ is equal to NULB; otherwise, $j$ is equal to $N$. For a specific example, the NULB of scan cell 10 is four, so $j=\mathrm{NULB}=4$. Equation (5) states that, if NDUB of scan cell $i$ is available, then $k$ is equal to NDUB +1 ; otherwise, $k$ is equal to zero.

For example, the NDUB of scan cell 10 is zero, so $k=$ $\mathrm{NDUB}+1=1$

$$
\begin{aligned}
& j= \begin{cases}\text { NULB }, & \text { if NULB available } \\
N, & \text { if NULB unavailable }\end{cases} \\
& k= \begin{cases}\text { NDUB }+1, & \text { if NDUB available } \\
0, & \text { if NDUB unavailable. }\end{cases}
\end{aligned}
$$

\section{UB Jump Simulation}

There are two types of Jump Simulations: UB Jump Simulation and LB Jump Simulation. The former locates upper bounds and the latter locates lower bounds of every fault. They share the same principle except how ChUD-SI is filled. This section describes UB Jump Simulation and the coming Section III-E describes LB Jump Simulation.

There are $W-1$ jump bits in a single Jump Simulation, where $W$ stands for CPU word size. Jump bits earn their name because they leap from $\mathrm{LB}_{n}$ to $\mathrm{UB}_{n}$ in an equal distance. The position of the $w_{\text {th }}$ jump bit between $\mathrm{UB}_{n}$ and $\mathrm{LB}_{n}$ is calculated in the following equation:

$$
\begin{aligned}
& \text { jump } \operatorname{bit}(w)=\left\lfloor\frac{\mathrm{UB}_{n}-\mathrm{LB}_{n}+1}{W} \times w\right\rfloor \\
& +\mathrm{LB}_{n}, 1 \leq w \leq(W-1) .
\end{aligned}
$$

For simplicity, consider only one single fault for now. In UB Jump Simulation, the fault is assumed upstream to the jump 


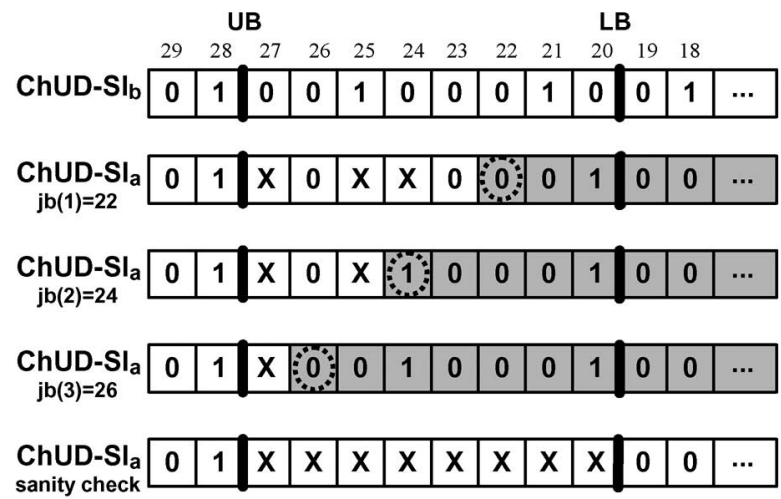

:- jump bits are circled

Fig. 7. UB Jump Simulation example ( $W=4$, HT fault).

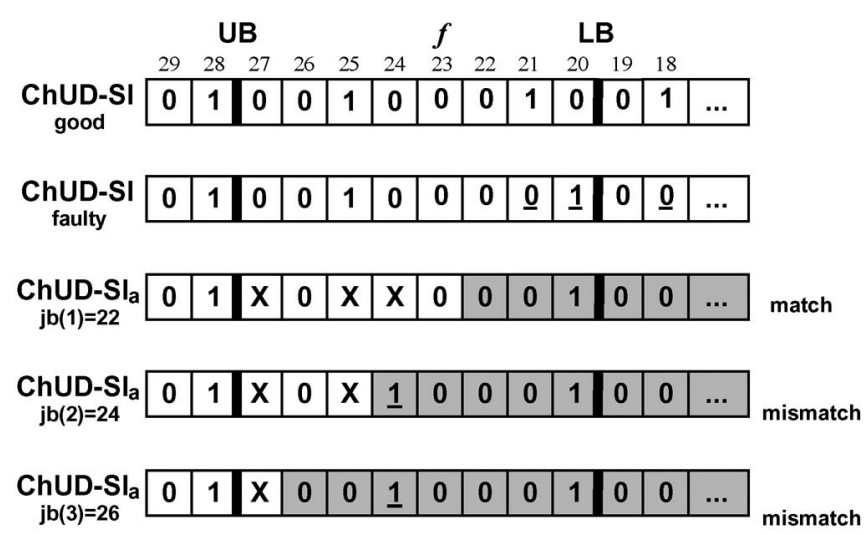

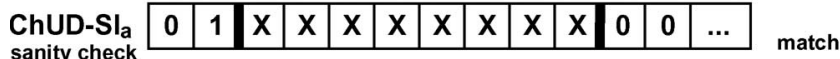

Fig. 8. UB updated to cell 23.

bit. If the assumption is correct, then affected bits between LB and the jump bit must be flipped, including the jump bit itself. However, affected bits between the UB and the jump bit are replaced by $\mathrm{X}$ (unknown) because the exact fault location is unknown. Consider the example of one single HT fault in Fig. 7. The original SI pattern is shown in the first row as ChUD - $\mathrm{SI}_{\mathrm{b}}$. Suppose that $W=4$ and four simulations are performed in parallel at the same time. Three jump bits (circled) leap in an equal distance from 22,24 , to 26 . Scan cells downstream to jump bits are painted in gray where affected bits are flipped. On the contrary, affected bits upstream to jump bits are replaced by X. Please note that scan cells 26 and 23 are filled with zeros because their values are not flipped in the presence of a single fault.

Fig. 8 shows how the upper bound is updated by UB Jump Simulation. Assume that the HT fault is located in cell 23. Affected bits in scan cells 21, 20, and 18 are flipped and underlined in ChUD - $\mathrm{SI}_{\text {faulty }}$. For jump bit 22, the simulation outputs $\left(\mathrm{OUT}_{\mathrm{S}}\right)$ match the observed outputs $\left(\mathrm{OUT}_{\mathrm{o}}\right)$ because there is no conflict between the faulty chain and the ChUD $-\mathrm{SI}_{\mathrm{a}}$. $\mathrm{OUT}_{\mathrm{S}}$ matches $\mathrm{OUT}_{\mathrm{O}}$ if every one and zero in $\mathrm{OUT}_{\mathrm{S}}$ is actually observed as one and zero in $\mathrm{OUT}_{\mathrm{O}}$, respectively. Don't care bits (Xs) in $\mathrm{OUT}_{\mathrm{s}}$ are not considered in the comparison. For jump bits 24 and 26, on the contrary, the simulation outputs may not match the observed outputs because of the difference between

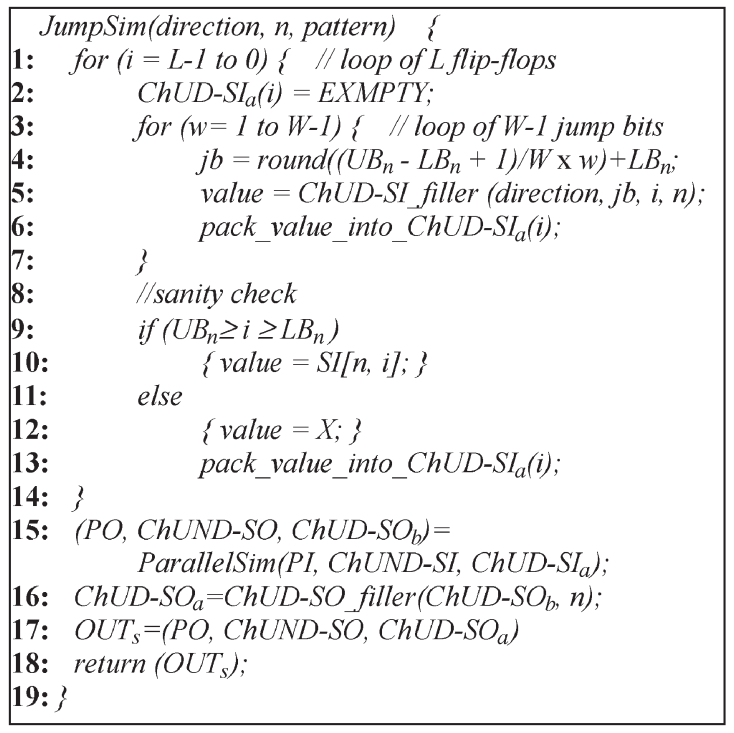

Fig. 9. Jump Simulation algorithm.

ChUD - $\mathrm{SI}_{\text {faulty }}$ and ChUD - $\mathrm{SI}_{\mathrm{a}}$. OUT $\mathrm{s}$ mismatches $\mathrm{OUT}_{\mathrm{o}}$ if any one or zero in $\mathrm{OUT}_{\mathrm{S}}$ is observed as an opposite value in $\mathrm{OUT}_{\mathrm{o}}$. If mismatch does occur in jump bit 24, it indicates that the fault is actually downstream to the jump bit. The upper bound is updated to scan cell 23, next to the most downstream mismatched jump bit. Please note that mismatched output is a sufficient condition but not a necessary condition. If mismatch occurs, the fault must be downstream to the jump bit. However, the statement is not true vice versa.

Please note that the last simulation is reserved for a sanity check where all affected bits between UB and LB are filled with $\mathrm{X}$. The sanity check is for detecting intermittent faults during diagnosis. If all faults are permanent and deterministic, the simulation outputs of sanity check should always match the observed outputs. If mismatches occur, there could exist intermittent faults such that UB or LB is incorrectly tightened during diagnosis. Accurate diagnosis of intermittent fault using Jump Simulation is still under research.

In the presence of multiple faults, the principle is still the same except that one UB Jump Simulation is performed for every fault. Fig. 9 shows the UB Jump Simulation algorithm for a given fault $f_{n}$. This algorithm contains two nested loops. The outer loop goes through $L$ scan cells and the inner loop goes through $W-1$ jump bits. After packing all ChUD-SI into a word, a parallel simulation is executed. Parallel fault simulation with unknowns can be found in previous publications, such as [20]. After the parallel simulation, the ChUD-SO filler masks those SOs that cannot be used for comparison. Finally, simulation outputs $\mathrm{OUT}_{\mathrm{s}}$ (including PO, ChUD - $\mathrm{SO}_{\mathrm{a}}$, and ChUND$\mathrm{SO})$ are returned to Bounds Finder.

The most critical part in Fig. 9 is the ChUD-SI filler, which determines the value of every scan cell $i$. The ChUD-SI filler algorithm in Fig. 10 is explained line by line using the example in Fig. 6. In lines 1-2 of the algorithm, both NULB, NDUB and $j, k$ are calculated for every scan cell $i$. In line 3 , if the potential values of cell $i$ are the same $(\mathrm{SI}[j, i]=\cdots=\mathrm{SI}[k, i])$, then its value is independent of the fault locations. The algorithm simply fills scan cell $i$ with the definite value, SI $[j, i]$. In Fig. 6, 


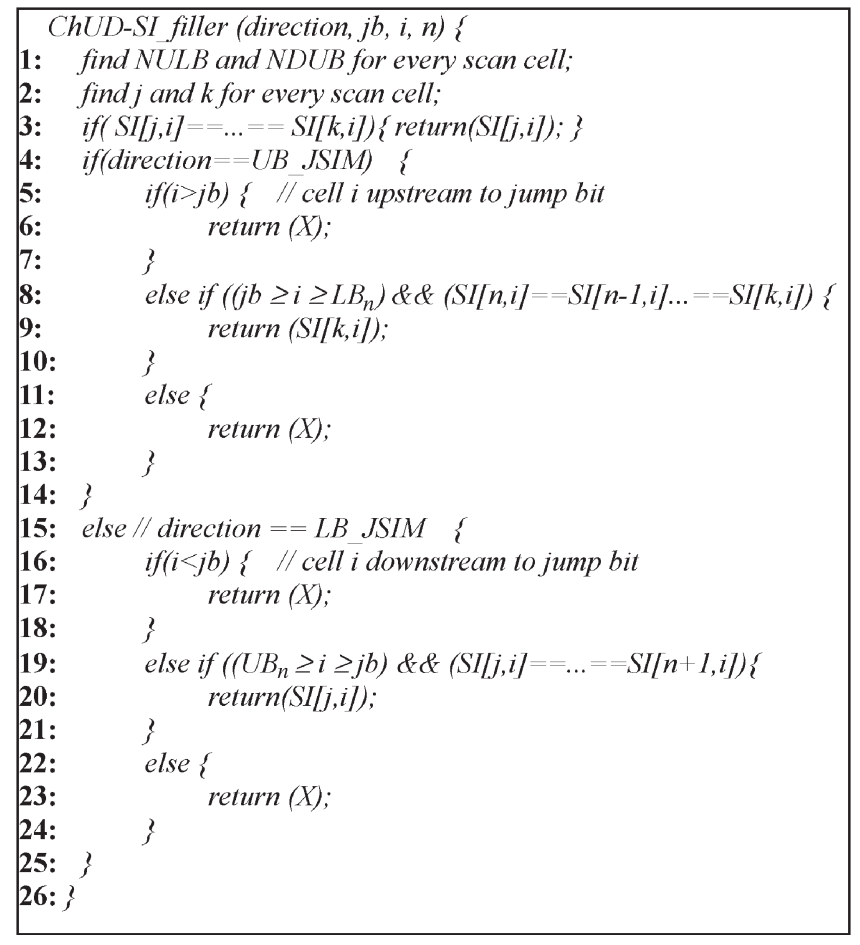

Fig. 10. ChUD-SI filler.

scan cell 6 is always zero $(\mathrm{SI}[2,6]=\mathrm{SI}[1,6]=0)$, so it is filled with zero all the time. The same rule applies to scan cells 15 , $14,5,4,3,2,1$, and 0 .

Continue on line 5 of the algorithm. If scan cell $i$ does not have a definite value and it is located upstream to the jump bit, then it is filled with X. For the same example in Fig. 6, assume that we are now filling ChUD-SI for fault $f_{2}(n=2)$, and the jump bit is cell $10(\mathrm{jb}=10)$. Scan cell 13 is upstream to the jump bit, and its potential values are not the same $(\mathrm{SI}[4,13] \neq$ $\mathrm{SI}[3,13])$. Scan cell 13 is filled with $\mathrm{X}$ because its exact values depend on the location of $f_{3}$. The same rule applies to scan cells $17,16,12$, and 11 .

Continue on line 8 of the algorithm. If scan cell $i$ does not have a definite value and it is downstream to the jump bit, then one more condition has to be checked. If the values from $\mathrm{SI}[n, i]$ to $\mathrm{SI}[k, i]$ are the same, scan cell $i$ can be safely filled with $\mathrm{SI}[n, i]$. Scan cell 9 is filled with zero because SI $[2,9]$ and SI $[1,9]$ are both zeros. We do not have to worry about the other value $\operatorname{SI}[3,9]=1$. Although $f_{3}$ is located in cell 9 and its actual value is 1 , we will encounter mismatches between $\mathrm{OUT}_{\mathrm{S}}$ and $\mathrm{OUT}_{\mathrm{o}}$. The consequence is that new $\mathrm{UB}_{2}$ will be updated to cell 9 , which is still a correct upper bound of $f_{2}$. The same rule also applies to scan cell 10.

Continue on line 12 of the algorithm, if none of the above conditions is met, scan cell $i$ is filled with X. Scan cell 8 has two different potential values: $\mathrm{SI}[2,8]=1, \mathrm{SI}[1,8]=0$. If scan cell 8 is filled with one and mismatch occurs, it is unclear whether $f_{2}$ is downstream or upstream to the jump bit. Therefore, scan cell 8 is filled with $\mathrm{X}$ and so is scan cell 7 .

Fig. 11 shows the algorithm to update UB. Simulation outputs $\mathrm{OUT}_{\mathrm{S}}$ are compared with observed outputs $\mathrm{OUT}_{\mathrm{O}}$. Please note that only the trustable ChUD-SO are considered, and the nontrustable ChUD-SO are ignored. How the trustable

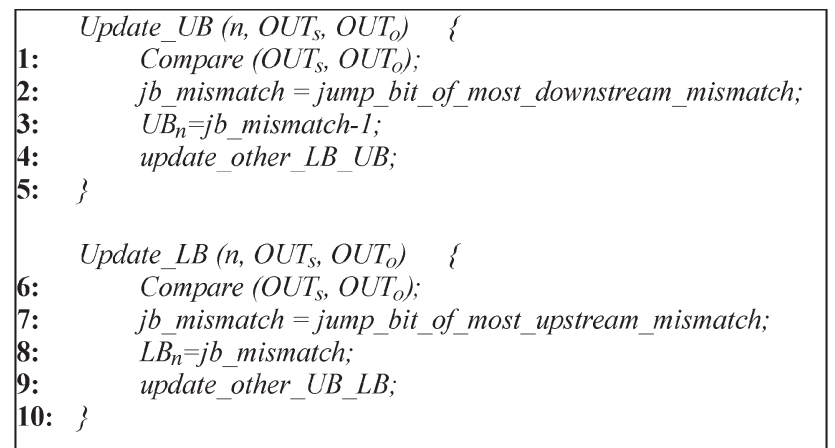

Fig. 11. Update UB and LB.

ChUD-SO is determined will be described in the next section. Any mismatch in the above three comparison (PO, ChUND-SI, ChUD-SI) indicates that the jump bit is actually good so the fault should be located in the downstream of the jump bit. New $\mathrm{UB}_{n}$ is relocated to the immediate downstream of the jump bit where the most downstream mismatch occurs. Finally, the other UB and LB are updated according to (1), (2), and (3), such that the relative orders of UB and LB are preserved.

\section{ChuD-SO Filler}

ChUD-SO can provide valuable diagnosis information if we know exactly which bits are trustable. Trustable ChUD-SO are those SOs in OUT ${ }_{\mathrm{s}}$ whose value do not depend on the fault locations. Trustable ChUD-SO can be used when comparing $\mathrm{OUT}_{\mathrm{S}}$ and $\mathrm{OUT}_{\mathrm{o}}$; the untrustable ChUD-SO are not compared.

The potential scan-out values of ChUD can be represented by an SO matrix. The SO matrix has $N+1$ rows and $L$ columns. Every element in this matrix is indexed by a fault index $(n)$ and a scan cell index (i). Fig. 12 shows an example of the SO matrix (HT fault, $N=5$ ). The first row $\mathrm{SO}[0, i]$ is the expected SOs without being affected by any fault. Every row in the SO matrix is the right shifted version of its preceding row. For $n$ greater than zero, the $n_{\text {th }}$ row of the SO matrix represents the SOs when the faulty chain is affected by $f_{n-1}$ only but not $f_{n}$.

The SO matrix for the other fault models can be derived in a similar way. Please note that the rows of the SO matrix are sorted in increasing order of $n$, which is opposite to the SI matrix.

To facilitate the subsequent description, two new bounds are defined. The SO upper bound of fault $f_{n}$ (denoted as $\mathrm{UB}_{n}^{*}$ ) is the most upstream scan cell of which the SO can be affected by fault $f_{n}$. $\mathrm{UB}_{n}^{*}=i$ means that fault $f_{n}$ can flip the SO of cell $i$ but not $i+1$. Similarly, the SO lower bound of fault $f_{n}\left(\mathrm{LB}_{n}^{*}\right)$ is the most downstream scan cell of which the can output can be affected by fault $f_{n}$. LB ${ }_{n}^{*}=i$ means that fault $f_{n}$ can flip the SO of cell $i$ but not $i-1$. The $\mathrm{UB}^{*}$ and $\mathrm{LB}^{*}$ can be calculated from the original UB and LB using the following two equations:

$$
\begin{aligned}
\mathrm{UB}_{n}^{*} & = \begin{cases}\mathrm{UB}_{n}+n, & \text { for ST/STR/STF faults } \\
\mathrm{UB}_{n}-n, & \text { for HT/FTR/FTF faults }\end{cases} \\
\mathrm{LB}_{n}^{*} & = \begin{cases}\mathrm{LB}_{n}+n, & \text { for ST/STR/STF faults } \\
\mathrm{LB}_{n}-n, & \text { for HT/FTR/FTF faults. }\end{cases}
\end{aligned}
$$

For slow-to faults, $\mathrm{UB}_{n}^{*}$ and $\mathrm{LB}_{n}^{*}$ are shifted upstream by $n$ from the original $\mathrm{UB}_{n}$ and $\mathrm{LB}_{n}$, respectively. For fast-to faults, 


\begin{tabular}{|c|c|c|c|c|c|c|c|c|c|c|c|c|c|c|c|c|c|c|}
\hline$i$ & 17 & 16 & 15 & 14 & 13 & 12 & 11 & 10 & 9 & 8 & 7 & 6 & 5 & 4 & 3 & 2 & 1 & 0 \\
\hline$S O[0, \mathrm{i}]$ & 0 & 1 & 1 & 1 & 1 & 1 & 0 & 0 & 0 & 0 & 1 & 0 & 0 & 1 & 0 & 1 & 1 & 0 \\
\hline$S O[1, \mathrm{i}]$ & X & 0 & 1 & 1 & 1 & 1 & 1 & 0 & 0 & 0 & 0 & 1 & 0 & 0 & - & - & - & - \\
\hline$S O[2, i]$ & - & $X$ & 0 & 1 & 1 & 1 & 1 & 1 & 0 & 0 & 0 & 0 & 1 & 0 & 0 & - & - & - \\
\hline$S O[3, i]$ & - & - & $\mathrm{X}$ & 0 & 1 & 1 & 1 & 1 & 1 & 0 & 0 & 0 & 0 & 1 & 0 & 0 & - & - \\
\hline$S O[4, i]$ & - & - & - & $X$ & 0 & 1 & 1 & 1 & 1 & 1 & 0 & 0 & 0 & 0 & 1 & 0 & 0 & - \\
\hline$S O[5, i]$ & - & - & - & - & $X$ & 0 & 1 & 0 & 1 & 1 & 1 & 0 & 0 & 0 & 0 & 1 & 0 & 0 \\
\hline UB & $\mathrm{UB}_{4}$ & & & & $\mathrm{UB}_{3}$ & $\mathrm{UB}_{2}$ & $\mathrm{UB}_{1}$ & & & & & & & & $\mathrm{UB}_{0}$ & & & \\
\hline $\mathrm{UB}^{*}$ & & & & & $\mathrm{UB}_{4}{ }^{*}$ & & & $\mathrm{UB}_{3,2,1^{*}}$ & & & & & & & $\mathrm{UB}_{0}{ }^{*}$ & & & \\
\hline LB & & & $\mathrm{LB}_{4}$ & & & & & & $\mathrm{LB}_{3}$ & & & $\mathrm{LB}_{2}$ & $\mathrm{LB}_{1}$ & & $\mathrm{LB}_{0}$ & & & \\
\hline $\mathrm{LB}^{*}$ & & & & & & & $\mathrm{LB}_{4}{ }^{*}$ & & & & & $\mathrm{LB}_{3}{ }^{*}$ & & $\mathrm{LB}_{2,1}{ }^{*}$ & $\mathrm{LB}_{0}{ }^{*}$ & & & \\
\hline$N U L B^{*}$ & - & - & - & - & - & - & 4 & 4 & 4 & 4 & 4 & 3 & 3 & 1 & 0 & 0 & 0 & 0 \\
\hline$N D U B^{*}$ & 4 & 4 & 4 & 4 & 3 & 3 & 3 & 0 & 0 & 0 & 0 & 0 & 0 & 0 & 0 & - & - & - \\
\hline$j^{*}$ & 5 & 5 & 5 & 5 & 5 & 5 & 4 & 4 & 4 & 4 & 4 & 3 & 3 & 1 & 0 & 0 & 0 & 0 \\
\hline$k^{*}$ & 5 & 5 & 5 & 5 & 4 & 4 & 4 & 1 & 1 & 1 & 1 & 1 & 1 & 1 & 0 & 0 & 0 & 0 \\
\hline ChUD-SOa & $\mathrm{u}$ & $\mathrm{u}$ & $\mathrm{u}$ & $\mathrm{u}$ & $\mathrm{u}$ & $\mathrm{u}$ & 1 & $\mathrm{u}$ & $\mathrm{u}$ & $\mathrm{u}$ & 0 & $\mathrm{u}$ & $\mathrm{u}$ & 0 & 0 & 1 & 1 & 0 \\
\hline
\end{tabular}

Fig. 12. SO matrix and trustable ChUD-SO.

$\mathrm{UB}_{n}^{*}$ and $\mathrm{LB}_{n}^{*}$ are shifted downstream by $n$ from the original $\mathrm{UB}_{n}$ and $\mathrm{LB}_{n}$. In Fig. 12, the original bounds of $f_{4}$ are $\mathrm{UB}_{4}=$ 17 and $\mathrm{LB}_{4}=15$. According to (4) and (5), $\mathrm{UB}_{4}^{*}=\mathrm{UB}_{4}-4=$ 13 and $\mathrm{LB}_{4}^{*}=\mathrm{LB}_{4}-4=11$. This means that fault $f_{4}$ can affect the SOs of scan cells 13,12, and 11. Please note that $\mathrm{UB}_{3}^{*}, \mathrm{UB}_{2}^{*}$, and $\mathrm{UB}_{1}^{*}$ are located in the same scan cell. $\mathrm{UB}^{*}$ and $\mathrm{LB}^{*}$ do not have to obey the monotonic increasing rules in (1) and (2).

Similar to the SI matrix, the potential value of a scan cell $i$ form a continuous segment in the $\mathrm{SO}$ matrix, from $\mathrm{SO}\left[j^{*}, i\right]$ to $\mathrm{SO}\left[k^{*}, i\right]$. Variable $j^{*}$ represents the maximum fault index and variable $k^{*}$ represents the minimum fault index. $j^{*}$ and $k^{*}$ are calculated from (9) and (10), which are similar to (4) and (5). NULB $^{*}$ is the smallest fault index of $\mathrm{LB}^{*}$ upstream to cell $i$, including cell $i$ itself. NDUB* is the largest fault index of UB* downstream to cell $i$, excluding cell $i$ itself

$$
\begin{aligned}
& j^{*}= \begin{cases}\mathrm{NULB}^{*}, & \text { if } \mathrm{NULB}^{*} \text { available } \\
N, & \text { if NULB}\end{cases} \\
& k^{*}= \begin{cases}\mathrm{NDUB}^{*}+1, & \text { if NDUailable } \\
0, & \text { if NDUB }\end{cases}
\end{aligned}
$$

For every scan cell, the potential SOs between $j^{*}$ and $k^{*}$ are highlighted in the SO matrix in Fig. 12. For the instance of scan cell $11, j^{*}=\mathrm{NULB}^{*}=4$ and $k^{*}=\mathrm{NDUB}^{*}+1=4$. Because $j^{*}=k^{*}=4$, the SO of scan cell 11 must be one, regardless of the other fault locations. The SO of scan cell 11 is therefore trustable. Totally, there are six trustable ChUD-SO: scan cells 11, 7, 4, 3, 2, and 1. Consider scan cell 9 as another example. $\mathrm{NULB}^{*}=4, \mathrm{NDUB}^{*}=0$ and $j^{*}=4, k^{*}=1$. Because the four potential values are different, the SO of scan cell 9 depends on the other fault locations. It is not trustable, and it is indicated by a " $u$ " symbol in the figure. Totally, there are 11 untrustable bits in the SOs. The ChUD-SO filler can be implemented in a similar to ChUD-SI filler, and hence, the details are skipped.

\section{E. LB Jump Simulation}

LB Jump Simulation is similar to UB Jump Simulation except for the way how affected bits are filled. In LB Jump Simulation, the fault is assumed downstream to the jump bit.

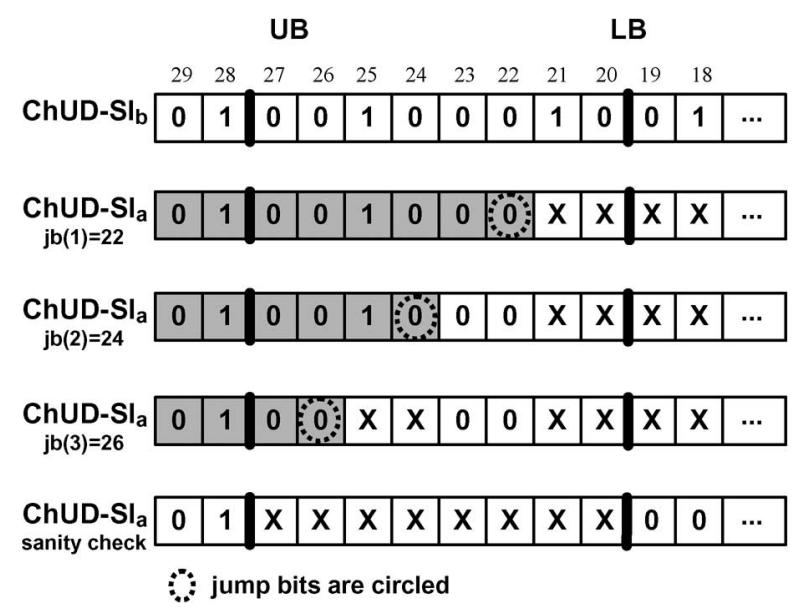

Fig. 13. LB Jump Simulation example.

Fig. 13 shows an example of LB Jump Simulation of a single HT fault, whose $\mathrm{UB}=27$ and $\mathrm{LB}=20$. The affected bits upstream to the jump bit remain unchanged, including the jump bit itself. The affected bits downstream to the jump bit are filled with Xs, excluding the jump bit itself. Please note that the jump bit itself is now good in the LB Jump Simulation. After the LB Jump Simulation, OUT ${ }_{s}$ is compared with $\mathrm{OUT}_{\mathrm{O}}$. A mismatch indicates that the assumption is wrong so the fault must be upstream to the jump bit. The new LB is relocated to the jump bit where the most upstream mismatch occurs. For the same example in Fig. 9, if the simulation outputs of jump bits 22 mismatch the observed outputs, then, the new LB becomes 22 .

The lower half of Fig. 10 shows the ChUD-SI filling algorithm for LB Jump Simulation in the presence of multiple faults. In lines 15-18, if a scan cell does not have a definite value and it is located downstream to the jump bit, then it is filled with $\mathrm{X}$. The last row in Fig. 6 demonstrates such an example. Suppose that $n=2$ and $\mathrm{jb}=10$. Scan cells 9, 8, and 7 are downstream to the jump bit so they are filled with X. In lines 19-21, if a scan cell is upstream to the jump bit, then one more condition has to be checked. If the values from $\mathrm{SI}[j, i]$ to $\mathrm{SI}[n+1, i]$ are the same, scan cell $i$ is filled with the complement of SI $[n, i]$. For example, scan cell 12 is filled with zero because $\mathrm{SI}[4,12]=$ $\mathrm{SI}[3,12]=0$. If we encounter mismatch between $\mathrm{OUT}_{\mathrm{s}}$ and 


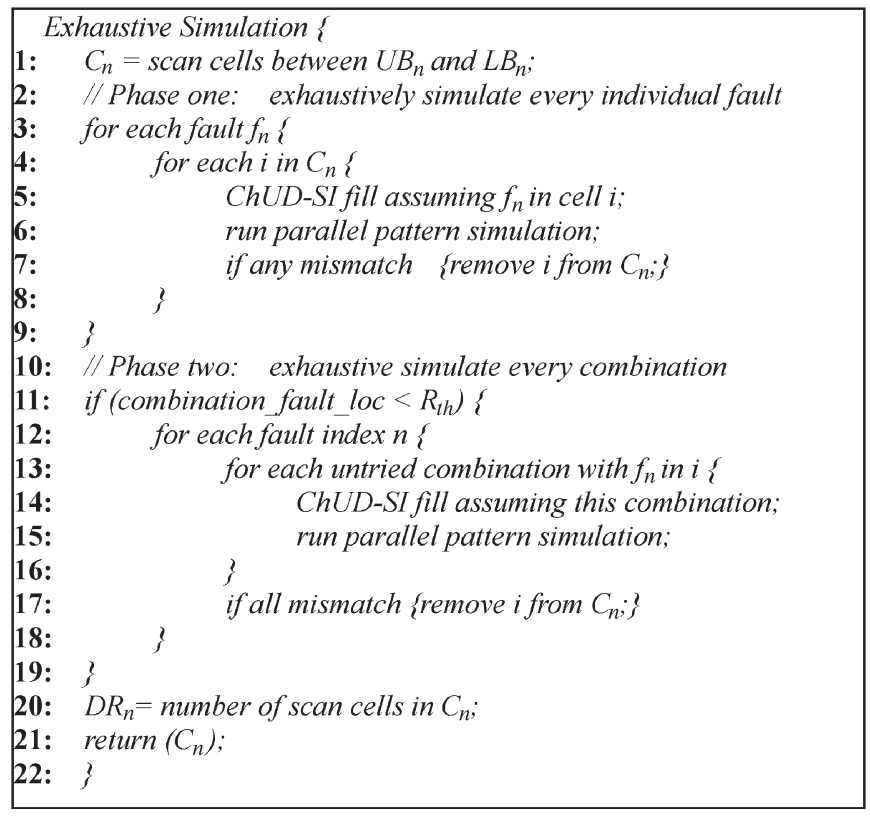

Fig. 14. Exhaustive simulation.

$\mathrm{OUT}_{\mathrm{o}}$, then scan cell 12 is actually one. $f_{2}$ is upstream to scan cell 12 so new $\mathrm{LB}_{2}$ can be moved to scan cell 12. The same rule applies to scan cell 11 .

\section{F. Exhaustive Simulation}

In exhaustive simulation, every scan cell between $\mathrm{UB}_{n}$ and $\mathrm{LB}_{n}$ is further examined in detail. The exhaustive fault simulation is divided into two phases: 1) exhaustive simulation for each individual fault and 2) exhaustive simulation for each combination of fault locations. In the first phase, every fault is simulated separately, independent of the other faults. In the second phase, every combination of fault locations is simulated if possible. Finally, the candidate set of every fault is returned as the final diagnosis result. The candidate set of fault $f_{n}\left(C_{n}\right)$ is the set of scan cells, not necessarily continuous, that fault $f_{n}$ can be located. The diagnosis resolution of fault $f_{n}\left(\mathrm{DR}_{n}\right)$ is the number of scan cells in $C_{n}$. Fig. 14 shows the outline of the exhaustive simulation algorithm.

Lines 1-2. Initially, $C_{n}$ contains all scan cells between $\mathrm{UB}_{n}$ and $\mathrm{LB}_{n}$.

Lines 3-4. Phase one exhaustive simulation is a twolevel nested loop of every fault index $n$ and every scan cell $i$ in $C_{n}$.

Lines 5-9. Assume that fault $f_{n}$ is indeed in cell $i$. This is achieved by calling ChUD-SI filler with both $\mathrm{UB}_{n}$ and $\mathrm{LB}_{n}$ set to $i$. Perform a parallel simulation of $W$ patterns. If mismatch occurs in any pattern, scan cell $i$ is proven not faulty. Remove scan cell $i$ from $C_{n}$.

Lines 10-11. Phase two exhaustive simulation is executed only if the number of combinations is less than a predefined threshold $R_{\mathrm{th}}$.

Lines 12-13. For every fault index $n$ and every scan cell $i$ in $C_{n}$, find an untried combination of fault locations with $f_{n}$ in scan cell $i$.
Lines 14-16. Fill ChUD-SI and perform parallel simulation of $W$ patterns.

Lines 17-19. If mismatch occurs in every simulation of scan cell $i$, then scan cell $i$ is removed from $C_{n}$. That is, scan cell $i$ remains in $C_{n}$ as long as there exist at least one combination whose simulation outputs match the observed outputs.

Lines 21-22. Finally, calculate diagnosis resolution $\mathrm{DR}_{n}$ for every fault and return the candidate set.

\section{EXPERIMENTAL RESULTS}

To demonstrate the effectiveness of the proposed technique, experiments are performed on ISCAS' 89 benchmark circuits with single stuck-at fault test patterns applied. The pattern generator is a modified version of ATALANTA [14], which performs dynamic test compaction, so the test length is small. The scan flip-flops in every benchmark circuit are evenly partitioned into two scan chains. The following experiments assume only one single faulty chain. Because there is no silicon involved, the observed faulty outputs $\left(\mathrm{OUT}_{\mathrm{O}}\right)$ are actually produced by our scan chain fault simulator. The simulations are performed on 32-bit machines ( $W=32)$.

Table III shows the experimental results of Bounds Finder on HT fault. The first column shows the length of the faulty chain $(L)$, and the second column shows the number of test patterns $(P)$. The bound resolution of fault $f_{n}\left(\mathrm{BR}_{n}\right)$ is the number of scan cells between $\mathrm{UB}_{n}$ and $\mathrm{LB}_{n}$, i.e.,

$$
\mathrm{BR}_{n}=\mathrm{UB}_{n}-\mathrm{LB}_{n}+1 \text {. }
$$

For each benchmark circuit, 100 cases are performed. In every case, $N$ faults are randomly selected in the faulty chain. The average BR, on the left of the slash, is the mean of BR among $100 N$ faults. The worst BR, on the right of the slash, is the largest BR among $100 N$ faults. It can be observed that the average BR is generally very small, which means many faults can be easily diagnosed by Bounds Finder only.

Table IV shows the diagnosis resolution of HT fault after exhaustive simulation (continued from Table III). The average DR is the mean of DR among $100 N$ faults. The worst DR is the largest DR among $100 N$ faults. For all benchmark circuits, we are able to achieve nearly exact diagnosis, even in the presence of $16 \mathrm{HT}$ faults in a chain. Because Bounds Finder is very successful, the phase two exhaustive simulation is performed for every case. The results validate the effectiveness of the proposed diagnosis for multiple HT faults.

Table $\mathrm{V}$ shows the average and the worst DR of six fault models. One hundred cases are performed for each circuit, and $N$ faults are injected in a single faulty chain for each case. The average DR is mostly below three, except STR and FTF of s38417. On the average, each case can be diagnosed within 3 min on a Linux machine with a 3.4-GHz CPU. The diagnosis resolutions of the other four fault models are, in general, worse than those of HT and ST faults. This is because HT/ST faults are excited by both the rising and the falling transitions, but the other faults are excited by only 
TABLE III

BOUND RESOlUtion OF HT FAULT (AVERAGE/WORST)

\begin{tabular}{|l|l|c|l|l|l|l|l|c|}
\hline circuit & $L$ & $P$ & $N=1$ & $N=4$ & $N=7$ & $N=10$ & $N=13$ & $N=16$ \\
\hline $\mathrm{s} 13207$ & 319 & 255 & $1.3 / 4$ & $1.5 / 4$ & $1.5 / 4$ & $1.5 / 5$ & $1.5 / 5$ & $1.6 / 5$ \\
\hline $\mathrm{s} 15850$ & 267 & 132 & $1.1 / 2$ & $1.2 / 3$ & $1.2 / 3$ & $1.2 / 4$ & $1.2 / 4$ & $1.2 / 4$ \\
\hline $\mathrm{s} 35932$ & 864 & 148 & $1.0 / 1$ & $1.0 / 2$ & $1.0 / 1$ & $1.0 / 2$ & $1.1 / 3$ & $1.1 / 3$ \\
\hline $\mathrm{s} 38417$ & 818 & 670 & $1.9 / 25$ & $1.9 / 25$ & $1.8 / 25$ & $2.2 / 26$ & $2.3 / 26$ & $2.2 / 26$ \\
\hline $\mathrm{s} 38584$ & 713 & 480 & $1.0 / 2$ & $1.0 / 2$ & $1.0 / 2$ & $1.0 / 2$ & $1.0 / 2$ & $1.0 / 2$ \\
\hline
\end{tabular}

TABLE IV

Diagnosis Resolution of HT Fault (AvERAgE/Worst)

\begin{tabular}{|l|l|c|c|c|c|c|c|c|}
\hline circuit & $L$ & $P$ & $N=1$ & $N=4$ & $N=7$ & $N=10$ & $N=13$ & $N=16$ \\
\hline $\mathrm{s} 13207$ & 319 & 255 & $1.0 / 1$ & $1.0 / 2$ & $1.0 / 2$ & $1.0 / 3$ & $1.0 / 2$ & $1.0 / 2$ \\
\hline $\mathrm{s} 15850$ & 267 & 132 & $1.0 / 1$ & $1.0 / 1$ & $1.0 / 2$ & $1.0 / 1$ & $1.0 / 1$ & $1.0 / 1$ \\
\hline $\mathrm{s} 35932$ & 864 & 148 & $1.0 / 1$ & $1.0 / 2$ & $1.0 / 1$ & $1.0 / 2$ & $1.0 / 2$ & $1.0 / 2$ \\
\hline $\mathrm{s} 38417$ & 818 & 670 & $1.0 / 2$ & $1.0 / 2$ & $1.0 / 3$ & $1.0 / 3$ & $1.0 / 3$ & $1.0 / 4$ \\
\hline $\mathrm{s} 38584$ & 713 & 480 & $1.0 / 1$ & $1.0 / 2$ & $1.0 / 1$ & $1.0 / 1$ & $1.0 / 1$ & $1.0 / 1$ \\
\hline
\end{tabular}

TABLE V

Diagnosis Resolution of Six Fault Models (Average/Worst)

\begin{tabular}{|l|c|c|c|c|c|c|c|}
\hline Circuit & $\mathrm{N}$ & $\mathrm{HT}$ & $\mathrm{ST}$ & $\mathrm{STR}$ & $\mathrm{STF}$ & $\mathrm{FTR}$ & $\mathrm{FTF}$ \\
\hline $\mathrm{s} 13207$ & 1 & $1.3 / 4$ & $1.0 / 1$ & $1.0 / 1$ & $1.0 / 2$ & $1.0 / 2$ & $1.0 / 2$ \\
\cline { 2 - 8 } $\mathrm{L}=319$ & 4 & $1.5 / 4$ & $1.0 / 2$ & $1.1 / 4$ & $1.1 / 3$ & $1.2 / 4$ & $1.2 / 4$ \\
\cline { 2 - 8 } $\mathrm{P}=255$ & 7 & $1.5 / 4$ & $1.0 / 2$ & $4.0 / 23$ & $2.4 / 19$ & $2.4 / 14$ & $3.3 / 22$ \\
\hline $\mathrm{s} 15850$ & 1 & $1.1 / 2$ & $1.0 / 1$ & $1.0 / 1$ & $1.0 / 2$ & $1.0 / 2$ & $1.0 / 2$ \\
\cline { 2 - 8 } $\mathrm{L}=267$ & 4 & $1.2 / 3$ & $1.0 / 1$ & $1.1 / 3$ & $1.1 / 3$ & $1.1 / 2$ & $1.3 / 3$ \\
\cline { 2 - 8 } $\mathrm{P}=132$ & 7 & $1.2 / 3$ & $1.0 / 3$ & $5.1 / 33$ & $1.6 / 14$ & $2.0 / 17$ & $5.4 / 25$ \\
\hline $\mathrm{s} 35932$ & 1 & $1.0 / 1$ & $1.0 / 1$ & $1.0 / 1$ & $1.0 / 1$ & $1.0 / 2$ & $1.0 / 1$ \\
\cline { 2 - 8 } $\mathrm{L}=864$ & 4 & $1.0 / 2$ & $1.0 / 1$ & $1.0 / 1$ & $1.0 / 1$ & $1.0 / 1$ & $1.0 / 2$ \\
\cline { 2 - 8 } $\mathrm{P}=148$ & 7 & $1.0 / 1$ & $1.0 / 6$ & $1.6 / 9$ & $1.8 / 14$ & $1.7 / 9$ & $1.7 / 9$ \\
\hline $\mathrm{s} 38417$ & 1 & $1.9 / 25$ & $1.2 / 2$ & $1.1 / 2$ & $1.2 / 2$ & $1.1 / 2$ & $1.0 / 2$ \\
\cline { 2 - 8 } $\mathrm{L}=818$ & 4 & $1.9 / 25$ & $1.2 / 2$ & $1.3 / 25$ & $1.2 / 2$ & $1.5 / 29$ & $2.4 / 41$ \\
\cline { 2 - 8 } $\mathrm{P}=670$ & 7 & $1.8 / 25$ & $1.2 / 7$ & $7.3 / 54$ & $4.9 / 37$ & $8.0 / 38$ & $14.9 / 57$ \\
\hline $\mathrm{S} 38584$ & 1 & $1.0 / 2$ & $1.0 / 1$ & $1.0 / 1$ & $1.0 / 1$ & $1.0 / 1$ & $1.0 / 1$ \\
\cline { 2 - 7 } $\mathrm{L}=713$ & 4 & $1.0 / 2$ & $1.0 / 1$ & $1.0 / 2$ & $1.0 / 1$ & $1.0 / 2$ & $1.0 / 2$ \\
\cline { 2 - 7 } $\mathrm{P}=480$ & 7 & $1.0 / 2$ & $1.0 / 1$ & $1.2 / 4$ & $1.1 / 3$ & $1.1 / 4$ & $1.3 / 6$ \\
\hline
\end{tabular}

one transition. Given the same SI patterns, the probability of exciting HT/ST faults is higher than that of the other fault models.

After further investigation, it is also observed that the don't care filling of SI patterns has significant impact on the diagnosis results. The ATPG algorithm randomly fills don't care bits in the SI pattern. The neighbor of a zero is likely to be one because most bits are Xs. For example, the original test cube " $0 \mathrm{xx} 1 \mathrm{x}$ " can be randomly filled as "01010," which becomes all zeros "00000" in the downstream of an STR fault. Randomly filled test patterns are not ideal for diagnosis purpose because the ChUD - $\mathrm{SI}_{\mathrm{a}}$ would be very monotonic. The lack of diversity explains why the diagnosis results of the four fault models are worse than HT fault.

Fig. 15 shows the decreasing BR with the number of test patterns applied (s38584, ten HT faults). The upper curve represents the worst BR and the lower curve represents average DR. Each curve is the experimental result of 100 cases, each of which has ten HT faults randomly injected. The average BR is as low as 5.7 using only the first pattern, and the worst BR decreases rapidly in the first three patterns. Fig. 16 shows the decreasing BR with the number of simulations. A simulation

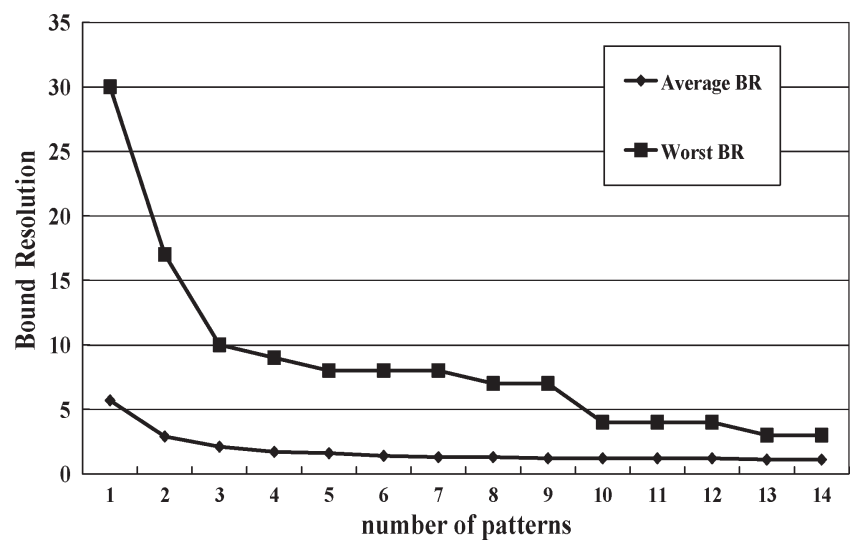

Fig. 15. BR versus number of patterns.

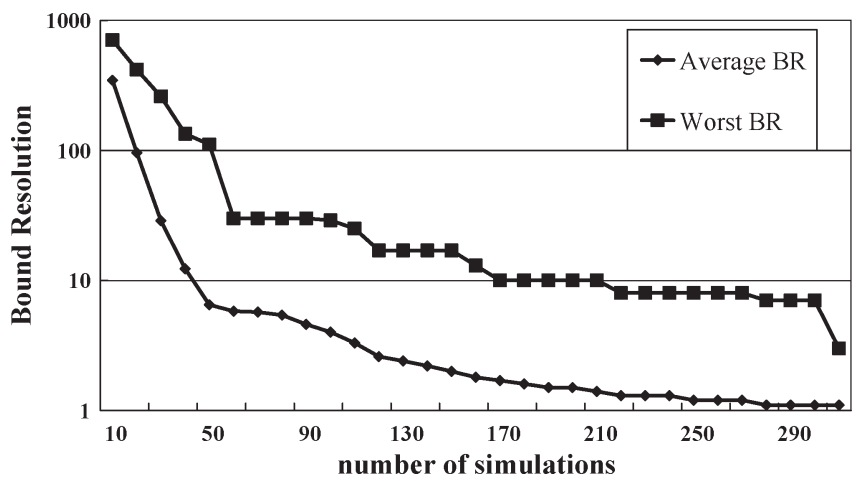

Fig. 16. BR versus number of simulations.

can be either a UB or an LB Jump Simulation of a single pattern. It can be seen that BR decreases rapidly in the first few simulations. Specifically, the average BR is as low as 5.8 in just 50 simulations. This means that many HT faults are quickly diagnosed using Bounds Finder only. In this experiment, test patterns are reordered randomly for every case to eliminate the bias of a particular pattern order.

The left portion of Table VI shows the average DR where only ten patterns are applied. This is to emulate the production 
TABLE VI

Average DR, Number of Simulations, ANd Run Time for Ten Patterns

\begin{tabular}{|c|c|c|c|c|c|c|c|c|c|c|c|c|c|}
\hline \multirow[t]{2}{*}{ circuit } & \multirow[t]{2}{*}{$N$} & \multicolumn{6}{|c|}{ Average Diagnosis Resolution } & \multicolumn{6}{|c|}{ Average Number of Simulation (run time in seconds) } \\
\hline & & HT & ST & STR & STF & FTR & FTF & HT & ST & STR & STF & FTR & FTF \\
\hline \multirow{3}{*}{$\begin{array}{l}\mathrm{s} 13207 \\
\mathrm{~L}=319 \\
\mathrm{P}=10\end{array}$} & 1 & 1.0 & 1.0 & 1.1 & 1.1 & 1.3 & 1.2 & $26(0.2)$ & $22(0.2)$ & $29(0.3)$ & $30(0.1)$ & $31(0.3)$ & $31(0.3)$ \\
\hline & 4 & 1.1 & 1.0 & 3.2 & 2.7 & 2.8 & 3.5 & $125(1.0)$ & $106(0.8)$ & $593(3.5)$ & $413(1.0)$ & $463(2.8)$ & 654 (3.6) \\
\hline & 7 & 1.1 & 1.0 & 12.7 & 9.4 & 10.3 & 13.1 & $254(2.1)$ & $208(1.7)$ & $601(4.9)$ & 613 (1.9) & $585(4.7)$ & $616(2.2)$ \\
\hline \multirow{3}{*}{$\begin{array}{l}S 15850 \\
L=267 \\
P=10\end{array}$} & 1 & 1.0 & 1.0 & 1.1 & 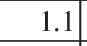 & 1.2 & 1.4 & $24(0.1)$ & $19(0.1)$ & $28(0.1)$ & $27(0.1)$ & $28(0.1)$ & $29(0.1)$ \\
\hline & 4 & 1.0 & 1.0 & 3.6 & 2.1 & 2.1 & 3.7 & $112(0.4)$ & $88(0.3)$ & $620(1.7)$ & $264(0.9)$ & $282(0.9)$ & $721(2.0)$ \\
\hline & 7 & 1.0 & & 15.8 & 7.5 & 7.8 & & $220(0.8)$ & $175(0.7)$ & $603(2.3)$ & $557(2.1)$ & $534(2.1)$ & $597 \quad(2.4$ \\
\hline \multirow{3}{*}{$\begin{array}{l}s 35932 \\
L=864 \\
P=10\end{array}$} & 1 & 1.1 & - & 1 & & 1.1 & 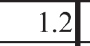 & $16(0.2)$ & $16(0.2)$ & $26(0.3)$ & $25(0.3)$ & $26(0.3)$ & $25(0.2)$ \\
\hline & 4 & 1.0 & 1.0 & 4.5 & 3.3 & 4.3 & 5.4 & 77 (0.6) & $80(0.7)$ & $489(3.5)$ & $555(3.7)$ & $573(3.8)$ & $609(4.3)$ \\
\hline & 7 & 1.1 & 1.0 & 25.5 & 20.3 & 18.2 & 25.0 & $164(1.5)$ & $156(1.5)$ & $769(7.4)$ & 607 (5.7) & $597(5.1)$ & 803 (7.9) \\
\hline \multirow{3}{*}{$\begin{array}{l}s 38417 \\
L=818 \\
P=10\end{array}$} & 1 & 1.5 & 1.2 & 1.4 & 1.6 & 2.4 & 2.8 & $33(0.3)$ & $30(0.3)$ & $38(0.6)$ & $38(0.5)$ & $41 \quad(0.5)$ & $41 \quad(0.5)$ \\
\hline & 4 & 2.5 & 1.2 & 15.7 & 8.9 & 3 & 17.9 & $320(2.5)$ & $176(1.6)$ & $664(6.3)$ & $647(5.7)$ & $673(5.8)$ & $610(5.7)$ \\
\hline & 7 & 4.5 & 1.3 & 36.0 & 21.0 & 21.4 & 38.2 & $770(6.1)$ & $540(4.5)$ & $1,300(14.6)$ & $1,011(13.2)$ & $1,080(12.2)$ & $1,283(14.3$ \\
\hline \multirow{3}{*}{$\begin{array}{l}\mathrm{s} 38584 \\
\mathrm{~L}=713 \\
\mathrm{P}=10\end{array}$} & 1 & 1.0 & 1.0 & 1.1 & & 1.2 & 1.3 & $22(0.3)$ & $15(0.2)$ & $27(0.3)$ & $25(0.3)$ & $27(0.3)$ & $30(0.4$ \\
\hline & 4 & 1.1 & 1.0 & 3.1 & 2.3 & 2.6 & 3.4 & $101(1.1)$ & $80(0.9)$ & $507(4.2)$ & $358(3.2)$ & $389(3.4)$ & $508(4.2)$ \\
\hline & 7 & 1.1 & 1.0 & 12.9 & 10.6 & 10.0 & 14.8 & $198(2.2)$ & $144(1.6)$ & $598(6.6)$ & $589(6.4)$ & $584(6.4)$ & $621 \quad 6.8$ \\
\hline
\end{tabular}

test environment where failure data are often truncated. The average DR is still very low for HT and ST faults. However, DR of the other fault models can be larger than desirable values. A possible remedy to this problem is to use a cycle-base ATE which collects more failure data than pin-base ATE [7]. A second solution is to apply special purpose diagnosis patterns in volume production.

The right portion of Table VI shows the average number of simulation (rounded to the nearest integer) and the average run time (in the parenthesis) for each diagnosis case. The run time is measured on a Linux system with $3.4-\mathrm{GHz}$ CPU. The average run time is less than $15 \mathrm{~s}$. The time complexity of this algorithm is case dependent. The number of simulations needed depends on many factors, such as fault locations, number of faults, chain length, test patterns, and the circuit structure. We provide a worst-case analysis as follows. For a single pattern, each fault would need no more than $L$ simulations to search for a bound. Therefore, total number of simulations is no more than $2 P L N$, where $P$ is the number of patterns, $L$ is chain length, and $N$ is number of faults. The factor of two is for UB and LB of each fault. Since a simulation can be performed in polynomial time, the overall diagnosis can be finished in polynomial time in the worst case.

To improve the diagnosis resolution in Table VI, an iterative experiment is performed. In every iteration, all test patterns are jump simulated to further refine the UB and LB. Table VII shows the average diagnosis resolution after the first and the third iteration. Only ten patterns are applied in this experiment. Because the Jump Simulation already did a good job in the first iteration, the improvement of iterative diagnosis is marginal.

In preceding experiments, faults are uniformly distributed in the faulty chain. It has been shown by past research that multiple defects tend to cluster in a small area [11]. Table VIII shows the average and the worst DR of clustered faults with all test patterns applied. In this experiment, $N$ faults are cluster in one tenth $(L / 10)$ of the faulty chain. The proposed technique still achieves an average DR better than three scan cells except
TABLE VII

Average Diagnosis Resolution of Ten Patterns (FIRST/THIRD ITERATION)

\begin{tabular}{|l|r|c|c|c|c|c|c|}
\hline circuit & N & HT & ST & STR & STF & FTR & FTF \\
\hline $\mathrm{s} 13207$ & 1 & $1.0 / 1.0$ & $1.0 / 1.0$ & $1.1 / 1.1$ & $1.1 / 1.1$ & $1.3 / 1.3$ & $1.2 / 1.2$ \\
\cline { 2 - 8 } $\mathrm{L}=319$ & 4 & $1.1 / 1.1$ & $1.0 / 1.0$ & $3.2 / 3.2$ & $2.7 / 2.7$ & $2.8 / 2.8$ & $3.5 / 3.5$ \\
\cline { 2 - 8 } $\mathrm{P}=255$ & 7 & $1.1 / 1.1$ & $1.0 / 1.0$ & $12.7 / 12.5$ & $9.4 / 9.2$ & $10.3 / 10.1$ & $13.1 / 12.8$ \\
\hline $\mathrm{S} 15850$ & 1 & $1.0 / 1.0$ & $1.0 / 1.0$ & $1.1 / 1.1$ & $1.1 / 1.1$ & $1.2 / 1.2$ & $1.4 / 1.4$ \\
\cline { 2 - 8 } $\mathrm{L}=267$ & 4 & $1.0 / 1.0$ & $1.0 / 1.0$ & $3.6 / 3.5$ & $2.1 / 2.1$ & $2.1 / 2.1$ & $3.7 / 3.7$ \\
\cline { 2 - 8 } $\mathrm{P}=132$ & 7 & $1.0 / 1.0$ & $1.0 / 1.0$ & $15.8 / 15.6$ & $7.5 / 7.4$ & $7.8 / 7.6$ & $15.7 / 15.6$ \\
\hline $\mathrm{S} 35932$ & 1 & $1.1 / 1.1$ & $1.0 / 1.0$ & $1.1 / 1.1$ & $1.0 / 1.0$ & $1.1 / 1.1$ & $1.2 / 1.2$ \\
\cline { 2 - 8 } $\mathrm{L}=864$ & 4 & $1.0 / 1.0$ & $1.0 / 1.0$ & $4.5 / 4.2$ & $3.3 / 3.2$ & $4.3 / 4.0$ & $5.4 / 4.7$ \\
\cline { 2 - 7 } $\mathrm{P}=148$ & 7 & $1.1 / 1.1$ & $1.0 / 1.0$ & $25.5 / 22.9$ & $20.3 / 17.6$ & $18.2 / 15.8$ & $25.0 / 20.2$ \\
\hline $\mathrm{S} 38417$ & 1 & $1.5 / 1.5$ & $1.2 / 1.2$ & $1.4 / 1.4$ & $1.6 / 1.6$ & $2.4 / 2.4$ & $2.8 / 2.8$ \\
\cline { 2 - 7 } $\mathrm{L}=818$ & 4 & $2.5 / 2.5$ & $1.2 / 1.2$ & $15.7 / 15.4$ & $8.9 / 8.8$ & $11.3 / 11.0$ & $17.9 / 17.8$ \\
\cline { 2 - 7 } $\mathrm{P}=670$ & 7 & $4.5 / 4.4$ & $1.3 / 1.3$ & $36.0 / 34.9$ & $21.0 / 20.6$ & $21.4 / 20.9$ & $38.2 / 37.2$ \\
\hline $\mathrm{s} 38584$ & 1 & $1.0 / 1.0$ & $1.0 / 1.0$ & $1.1 / 1.1$ & $1.0 / 1.0$ & $1.2 / 1.2$ & $1.3 / 1.3$ \\
\cline { 2 - 7 } $\mathrm{L}=713$ & 4 & $1.1 / 1.1$ & $1.0 / 1.0$ & $3.1 / 3.1$ & $2.3 / 2.3$ & $2.6 / 2.6$ & $3.4 / 3.4$ \\
\cline { 2 - 7 } $\mathrm{P}=480$ & 7 & $1.1 / 1.1$ & $1.0 / 1.0$ & $12.9 / 12.7$ & $10.6 / 10.4$ & $10.0 / 9.9$ & $14.8 / 14.4$ \\
\hline
\end{tabular}

TABLE VIII

Diagnosis Resolution of Clustered FAults (AvERAGE/Worst)

\begin{tabular}{|l|c|c|c|c|c|c|c|}
\hline circuit & $N$ & HT & ST & STR & STF & FTR & FTF \\
\hline $\mathrm{S} 13207$ & 1 & $1.0 / 3$ & $1.0 / 1$ & $1.0 / 1$ & $1.0 / 1$ & $1.0 / 2$ & $1.0 / 1$ \\
\cline { 2 - 8 } $\mathrm{L}=319$ & 4 & $1.0 / 2$ & $1.0 / 1$ & $1.1 / 4$ & $1.1 / 3$ & $1.1 / 4$ & $1.2 / 4$ \\
\cline { 2 - 7 } $\mathrm{P}=255$ & 7 & $1.0 / 2$ & $1.0 / 1$ & $1.6 / 7$ & $1.4 / 5$ & $1.7 / 5$ & $1.8 / 6$ \\
\hline $\mathrm{s} 15850$ & 1 & $1.0 / 1$ & $1.0 / 1$ & $1.0 / 1$ & $1.0 / 2$ & $1.0 / 1$ & $1.0 / 1$ \\
\cline { 2 - 7 } $\mathrm{L}=267$ & 4 & $1.0 / 1$ & $1.0 / 1$ & $1.1 / 3$ & $1.0 / 2$ & $1.1 / 3$ & $1.2 / 3$ \\
\cline { 2 - 7 } $\mathrm{P}=132$ & 7 & $1.0 / 1$ & $1.0 / 1$ & $1.8 / 6$ & $1.3 / 4$ & $1.4 / 6$ & $1.8 / 8$ \\
\hline $\mathrm{S} 35932$ & 1 & $1.0 / 1$ & $1.0 / 1$ & $1.0 / 1$ & $1.0 / 1$ & $1.0 / 1$ & $1.0 / 1$ \\
\cline { 2 - 7 } $\mathrm{L}=864$ & 4 & $1.0 / 2$ & $1.0 / 1$ & $1.0 / 1$ & $1.0 / 1$ & $1.0 / 2$ & $1.0 / 2$ \\
\cline { 2 - 7 } $\mathrm{P}=148$ & 7 & $1.0 / 2$ & $1.0 / 1$ & $1.0 / 1$ & $1.0 / 2$ & $1.0 / 3$ & $1.0 / 2$ \\
\hline $\mathrm{S} 38417$ & 1 & $1.0 / 2$ & $1.2 / 2$ & $1.2 / 2$ & $1.2 / 2$ & $1.0 / 2$ & $1.0 / 2$ \\
\cline { 2 - 7 } $\mathrm{L}=818$ & 4 & $1.0 / 2$ & $1.2 / 2$ & $1.2 / 2$ & $1.2 / 8$ & $1.2 / 8$ & $1.8 / 30$ \\
\cline { 2 - 7 } $\mathrm{P}=670$ & 7 & $1.0 / 2$ & $1.2 / 2$ & $3.0 / 37$ & $1.7 / 15$ & $2.0 / 30$ & $5.1 / 48$ \\
\hline $\mathrm{S} 38584$ & 1 & $1.0 / 1$ & $1.0 / 1$ & $1.0 / 1$ & $1.0 / 1$ & $1.0 / 1$ & $1.0 / 1$ \\
\cline { 2 - 7 } $\mathrm{L}=713$ & 4 & $1.0 / 1$ & $1.0 / 1$ & $1.0 / 1$ & $1.0 / 2$ & $1.0 / 2$ & $1.0 / 3$ \\
\cline { 2 - 7 } $\mathrm{P}=480$ & 7 & $1.0 / 1$ & $1.0 / 1$ & $1.1 / 4$ & $1.0 / 3$ & $1.1 / 3$ & $1.2 / 4$ \\
\hline
\end{tabular}

s38417. This experiment shows that the proposed technique is still effective to diagnose localized multiple scan chain faults. 


\section{Discussion}

\section{A. Multiple Faulty Chains}

The proposed Bounds Finder can be extended to diagnose multiple faults in multiple faulty chains using the divide and conquer approach. The idea is to diagnose one faulty chain at a time. First of all, the number of faults and the fault model of each faulty chain are determined using the two-pattern test in Table II. One chain is selected to be the ChUD; the other chains are ChUND. For each ChUD, an SI matrix is produced, and the ChUD-SI is filled in the same way as before. The SIs of faulty ChUND are filled with don't cares except those bits whose values do not change in the presence of faults. This filling approach prevents the interaction of faults among different faulty chains. After the Jump Simulation, every chain has its own SO matrix. All the SOs of good chains are observed. On the contrary, only the trustable SOs of faulty chains are observed. After one ChUD is diagnosed, a new faulty chain is chosen as ChUD, and the other chains are ChUND. The same procedure repeats until every faulty chain is diagnosed. The whole diagnosis can be iterated for several times until there is no more improvement in BR. The proposed approach is still applicable as long as the fault model in a faulty chain is the same.

\section{B. Inversions in Scan Chains}

For the ease of explanation, the above descriptions assume no inversion between scan cells. In the case of inversions in scan chains, two parts in the proposed diagnosis technique have to be modified. First, the pattern fillers have to fill correct values according to the parity of scan cells. Second, the observed SO has to be corrected by the parity of the scan chain. The other parts of the diagnosis algorithm remain mostly unchanged.

\section{Mixed Fault Models}

If all faults in a circuit are caused by a single systematic problem, they should belong to the same fault model. However, if this is not the case, there could be mixed fault models in a circuit. Two possible scenarios are discussed as follows. First, multiple chains are faulty, but all faults in a chain belong to the same fault model. The proposed technique is still applicable to this scenario, where each faulty chain is independently diagnosed in the way described in Section V-A. In the second scenario, faults in a faulty chain belong to more than one fault model. The proposed two-pattern test may not succeed in determining their fault models because two opposite fault models can cancel out each other. For example, flush test may not detect an STR fault and an FTR fault in the same chain because their effects cancel out.

\section{SUMMARY}

This paper presents a fast and precise algorithm to diagnose multiple timing faults in the scan chain. The novel Jump Simulation quickly identifies precise upper and lower bounds by using parallel simulation. Experimental results on ISCAS benchmark circuits show that nearly every one of 16 HT faults is exactly diagnosed. The proposed technique is still very effective even when the failure data are truncated due to limited ATE memory.

\section{ACKNOWLEDGMENT}

The authors would like to thank all reviewers for their comments.

\section{REFERENCES}

[1] S. Edirisooriya and G. Edirisooriya, "Diagnosis of scan path failures," in Proc. IEEE VLSI Test Symp., 1995, pp. 250-255.

[2] R. Guo and S. Venkataraman, "A technique for fault diagnosis of defects in scan chains," in Proc. IEEE Int. Test Conf., 2001, pp. 268-277.

[3] R. Guo and S. Venkataraman, "A new technique for scan chain failure diagnosis," in Proc. Int. Symp. Test. Failure Anal., 2002, pp. 723-732.

[4] J. Hirase, N. Shindou, and K. Akahori, "Scan chain diagnosis using IDDQ current measurement," in Proc. Asian Test Symp., 1999, pp. 153-157.

[5] Y. Huang, W.-T. Cheng, S. M. Reddy, C.-J. Hsieh, and Y.-T. Hung, "Statistical diagnosis for intermittent scan chain hold-time fault," in Proc. IEEE Int. Test Conf., 2003, pp. 319-327.

[6] Y. Huang, W.-T. Cheng, C.-J. Hsieh, H.-Y. Tseng, A. Huang, and Y.-T. Hung, "Intermittent scan chain fault diagnosis based on signal probability analysis," in Proc. IEEE Des. Autom. Test Eur. Conf., 2004, pp. 1530-1591.

[7] Y. Huang, W.-T. Cheng, N. Tamarapalli, J. Rajski, and R. Klingenberg, "Diagnosis with limited failure information," in Proc. Int. Test Conf., Oct. 2006, pp. 637-646.

[8] Y.-L. Kao, W.-S. Chuang, and C.-M. Li, "Jump simulation: A fast and precise scan chain fault diagnosis technique," in Proc. Int. Test Conf., Oct. 2006, pp. 1-9.

[9] J. Kibarian, "The nature of yield ramping: Keeping ahead of evolution," in Proc. IEEE Int. Test Conf., 2005, p. 12. keynote speech.

[10] B. Koenemann, "Test in the era of "What you see is NOT what you get,", in Proc. IEEE Int. Test Conf., 2004, p. 12. keynote speech.

[11] I. Koren, Z. Koren, and C. H. Stapper, "A statistical study of defect maps of large area VLSI IC's," IEEE Trans. Very Large Scale Integr. (VLSI) Syst., vol. 2, no. 2, pp. 249-256, Jun. 1994.

[12] B. Kruseman, A. Majhi, C. Hora, S. Eichenberger, and J. Meirlevede, "Systematic defects in deep sub-micron technologies," in Proc. IEEE Int. Test Conf., 2004, pp. 290-298.

[13] S. Kundu, "Diagnosing scan chain faults," IEEE Trans. Very Large Scale Integr. (VLSI) Syst., vol. 2, no. 4, pp. 512-516, Dec. 1994.

[14] H. K. Lee and D. S. Ha, "On the generation of test patterns of combinational circuits," Dept. Elect. Eng. Virginia Polytechnic Inst. State Univ., Blacksburg, VA, Tech. Rep., 12-93, 1991.

[15] J. C.-M. Li, C.-W. Tseng, and E. J. McCluskey, "Testing for resistive opens and stuck opens," in Proc. Int. Test Conf., Oct. 2001, pp. 1049-1058.

[16] J. C.-M. Li, "Diagnosis of single stuck-at faults and multiple timing faults in scan chains," IEEE Trans. Very Large Scale Integr. (VLSI) Syst., vol. 13, no. 6, pp. 708-718, Jun. 2005.

[17] J. C.-M. Li, "Diagnosis of multiple hold-time and setup-time faults in scan chains," IEEE Trans. Comput., vol. 54, no. 11, pp. 1467-1472, Nov. 2005.

[18] S. Narayanan and A. Das, "An efficient scheme to diagnose scan chains," in Proc. IEEE Int. Test Conf., 1997, pp. 704-713.

[19] P. Nigh, D. Vallett, A. Patel et al., "Failure analysis of timing and IDDq failures from the SEMATECH test methods experiment," in Proc. IEEE Int. Test Conf., 1998, pp. 43-52.

[20] D. Pradhan, Ed., Fault Tolerant Computing: Theory and Techniques. Englewood Cliffs, NJ: Prentice-Hall, 1986.

[21] J. Schafer, F. Policastri, and R. Mcnulty, "Partner SRLs for improved shift register diagnostics," in Proc. IEEE VLSI Test Symp., 1992, pp. 198-201.

[22] P. Song, F. Stellari, T. Xia, and A. Weger, "A novel scan chain diagnostics technique based on light emission from leakage current," in Proc. IEEE Int. Test Conf., 2004, pp. 140-147.

[23] K. Stanley, "High-accuracy flush-and-scan software diagnostic," in Proc. IEEE Des. Test. Comput., Nov./Dec. 2001, pp. 56-62.

[24] C.-W. Tzeng and S.-Y. Huang, "Diagnosis by image recovery: Finding mixed multiple timing faults in a scan chain," IEEE Trans. Circuits Syst. II, Exp. Briefs, vol. 54, no. 8, pp. 690-694, Aug. 2007.

[25] Y. Wu, "Diagnosis of scan chain failures," in Proc. Int. Symp. Defect Fault Tolerance VLSI Syst., 1998, pp. 217-222. 
[26] J.-S. Yang and S.-Y. Huang, "Quick scan chain diagnosis using signal profiling," in Proc. Int. Conf. Comput. Des., Oct. 2005, pp. 157-600.

[27] F. Motika, P. Nigh, P. Song, and H. B. Druckerman, "AC scan diagnostic method," U.S. Patent 6516432 B1, Feb. 4, 2003.

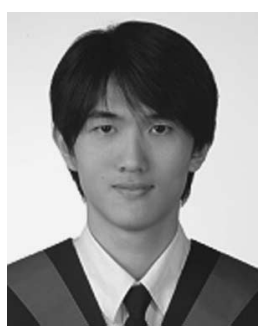

Wei-Shun Chuang received the B.S. degree in computer science and information engineering from the National Taiwan University, Taipei, Taiwan, R.O.C., in 2005, and the M.S. degree from the Graduate Institute of Electronics Engineering, National Taiwan University, in 2007.

$\mathrm{He}$ is currently an Engineer of Springsoft, Inc., Hsinchu, Taiwan. His research interest includes fault diagnosis.

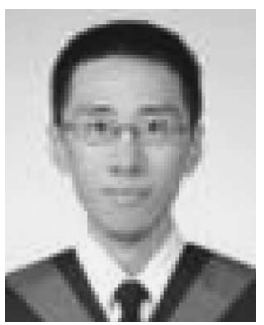

Shiu-Ting Lin was born in Changhua County, Taiwan, R.O.C., in 1984. He received the B.S. degree in electrical engineering from the National Taiwan University, Tapei, Taiwan, in 2006, where he has been working toward the M.S. degree in the Graduate Institute of Electronics Engineering since 2006.

His research interests include low-power testing and delay fault test.

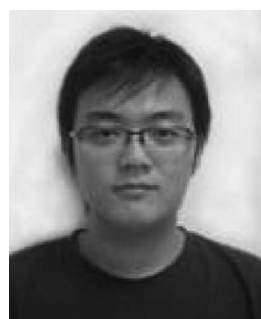

Wei-Chih Liu received the B.S. degree in computer science and information engineering from the National Taiwan University, Taipei, Taiwan, R.O.C., in 2006, where he is currently working toward the M.S. degree in the Graduate Institute of Electronics Engineering.

His research interests include fault diagnosis and physical design.

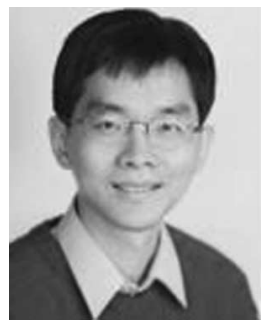

James Chien-Mo Li (M'02) received the B.S.E.E degree from the National Taiwan University, Taipei, Taiwan, R.O.C., in 1993, and the M.S.E.E. and $\mathrm{Ph} . \mathrm{D}$. degrees in electrical engineering from Stanford University, Stanford, CA, in 1997 and 2002, respectively.

$\mathrm{He}$ is currently an Associate Professor with the Graduate Institute of Electronics Engineering, National Taiwan University. His research interests include design for testability, built-in self test, lowpower testing, and fault diagnosis. 